\title{
Classification and Health Risk Assessment for Borehole Water Contaminated by Metals in Selected Households in Southwest Nigeria
}

\author{
Adeleke Adeniyi ${ }^{1 *}$, Kafeelah Yusuf ${ }^{1}$, Olumuyiwa Okedeyi ${ }^{2}$, Mutiu Sowemimo ${ }^{1}$ \\ ${ }^{1}$ Department of Chemistry, Lagos State University, Ojo, Nigeria \\ ${ }^{2}$ Department of Biochemistry, Lagos State University, Ojo, Nigeria \\ Email: "lekeadeniyi@yahoo.com
}

Received 17 February 2016; accepted 23 April 2016; published 26 April 2016

Copyright (C) 2016 by authors and Scientific Research Publishing Inc.

This work is licensed under the Creative Commons Attribution International License (CC BY).

http://creativecommons.org/licenses/by/4.0/

(c) (i) Open Access

\begin{abstract}
Samples of borehole water were randomly collected from twenty households in the Lagos-Ogun axis of southwestern Nigeria. The samples were analyzed for silver, iron, manganese, lead, nickel, magnesium, cadmium, zinc, sodium, potassium, $\mathrm{pH}$, turbidity, dissolved oxygen, electrical conductivity, alkalinity, total hardness, chloride, nitrate and sulphate following standard methods of water analysis. Results obtained were compared with global background values and WHO guidelines for drinking water. The $\mathrm{pH}$ values ranged from $4.17 \pm 0.26$ to $6.07 \pm 0.27$ while the metal pollution indexes are between 0.01 and 0.11 . Cadmium, manganese, iron and nitrate $(\mathrm{mg} / \mathrm{L})$ levels were found to be relatively high in most of the water samples. Results obtained were analyzed statistically. Health risk assessment for exposure to cancer and non-cancer indices was evaluated. Kempster and co-workers classification of drinking water quality was applied; nineteen of the twenty household water samples were found to fall short of the ideal water quality suitable for lifetime use.
\end{abstract}

\section{Keywords}

Borehole, Water Quality, Health Risk, Metals, Urbanization

\section{Introduction}

The unwholesomeness of drinking water resulting from contaminants released from different anthropogenic sources has become a global concern [1]-[4]. Rapid population growth, urbanization and seeming mismanagement

"Corresponding author.

How to cite this paper: Adeniyi, A., Yusuf, K., Okedeyi, O. and Sowemimo, M. (2016) Classification and Health Risk Assessment for Borehole Water Contaminated by Metals in Selected Households in Southwest Nigeria. Journal of Water Resource and Protection, 8, 459-471. http://dx.doi.org/10.4236/jwarp.2016.84039 
of resources have hampered the capacity of government owned municipal water providers to meet the needs of the populace [5]-[7], even in cases where community based boreholes are provided lack of maintenance make them go out of operation. In Nigeria, most household in urban and semi-urban areas rely on boreholes or shallow wells for their water supply. This is the situation in the Lagos-Ogun axis, where samples for the present study were collected. The deterioration of water quality can pose a high hazard to human health [8] [9]. To address this concern, water samples are expected to be routinely analyzed and measured against a range of health and non-health based physico-chemical standards. The majority of these standards are based on the World Health Organization (WHO) and United States Environmental Protection Agency (USEPA) guidelines [10].

There are global concerns that the usage of water above permitted limits could result to dire health consequences [6] [11]-[15]. These concerns are manifested in the significantly large number of metals ground water contamination studies across different countries. Representative global studies reviewed are from Nigeria, Malawi, Ghana, Ethiopia, Thailand, Iran, Pakistan, Bangladesh, Turkey, LAO PDR, Hong Kong, India and China [2] [9] [15]-[26].

In many countries of the world, there exist gaps in the provision of potable water. This challenge is even more exacerbated among developing countries like Nigeria with uncontrolled urbanization, population growth coupled with huge infrastructural deficits.

Consumption of unwholesome metal contaminated water has adverse health consequences [2]. This research work was undertaken to evaluate the wholesomeness of water sourced from boreholes in the selected LagosOgun States communities using WHO standards for drinking water as bench mark. The potential health risk occasioned by the exposure of inhabitants to metals contamination through water consumption was evaluated. Classification based on lifetime usage proposed by Kempster et al., 1997 [13] was adopted in this study.

\section{Materials and Methods}

\subsection{Study Area}

The sampling locations which are within the Lagos-Ogun State axis of Nigeria are indicated in Figure 1. These are predominantly densely populated urban and semi urban settlements. These communities are not covered by government owned municipal water supply network. In addition most of these communities indulge in unhealthy waste management practices like indiscriminate dumping of refuse in available open spaces and street corners and waste burning.

\subsection{Sampling and Sample Preparation}

Water samples were randomly collected from point of usage in the households fortnightly from July to December, 2013. One hundred groundwater samples were collected for physico-chemical analysis from twenty household boreholes located in the selected communities (Lagos locations are: Erunwen 1 (Yusuf), Erunwen 2 (Mama Tola), Erunwen 3 (Mama Azeezat), Agbele, Kokoro Abu, Eyita 1 (Jembi), Eyita 2 (Okedeyi), Eyita 3 (Ogunlana), Ikosi-Ketu, Oshodi, Ogudu, Akesan 1 (Okuneye), Akesan 2 (Ahove) and Ilado respectively; the Ogun locations are: Ogijo, Ibafo, Magboro, Ilupeju-Agbara, Lusada 1 (Taiwo) and Lusada 2 (Niyi). 2.5 L of water from each borehole were collected in sterile polypropylene bottles and analyzed for $\mathrm{pH}$, turbidity, dissolved oxygen, electrical conductivity, alkalinity, total hardness, chloride, nitrate and sulphate. Other $2.5 \mathrm{~L}$ samples of water from the same borehole were collected, acidified and analyzed for metals, silver (Ag), iron (Fe), manganese $(\mathrm{Mn})$, lead $(\mathrm{Pb})$, nickel $(\mathrm{Ni})$, magnesium $(\mathrm{Mg})$, cadmium $(\mathrm{Cd})$, zinc $(\mathrm{Zn})$, sodium $(\mathrm{Na})$ and potassium $(\mathrm{K})$ (Adeniyi et al. 2014; APHA, 1998).

\subsection{Analytical Procedure}

The $\mathrm{pH}$ was determined in situ using Jenway $3505 \mathrm{pH}$ meter; HACH - $2100 \mathrm{~N}$ Turbidity meter for turbidity; dissolved oxygen was determined by DO meter; Jenway 470 Conductivity meter for electrical conductivity; alkalinity, total hardness and chloride were determined by titrimetric methods; nitrate and sulphate were detected using HACH DR-3900. Whereas metals were evaluated using atomic absorption spectrophotometry with a Solar Thermo Electron Corporation AA Series Spectrometer. All analyses were carried out in accordance with standard methods for the examination of water and wastewater [27]. The results of the physico-chemical parameters 


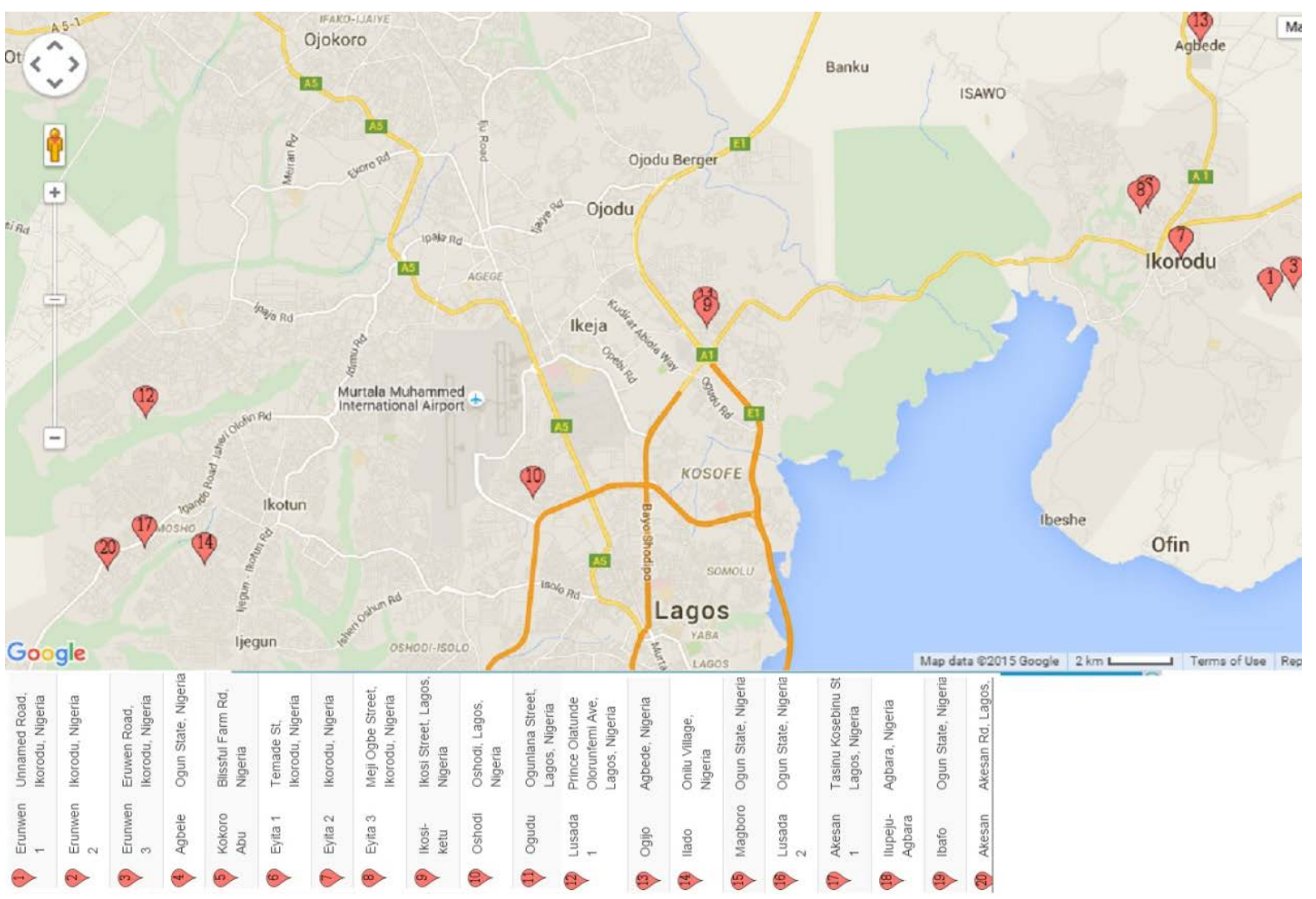

Figure 1. Global positioning system data (1 - 20 are the sampling locations).

and metal pollution index (MPI) are indicated in Table 1 and Table 2. The MPI is derived using the equation proposed by Usero et al., 1997 [28], as shown below:

$$
\text { MPI }=\left(C f_{1} \times C f_{2} \cdots C f_{n}\right)^{\frac{1}{n}}
$$

where, $C f_{n}=$ concentration of the metal $n$ in the sample.

\subsection{Quality Assurance}

All equipment was calibrated on daily basis using standard solutions. Samples were replicated; blank and previously spiked water samples were analyzed to further assure quality. Stock solutions (Merck) of $1000 \mathrm{mg} / \mathrm{L}$ of the different metals were used to prepare the calibration standards. The potential contamination of samples was evaluated by analyzing one acid blank in every batch [5] [29].

\subsection{Risk Assessment}

To assess potential health risks, interviews were conducted and questionnaires administered to members of the participating households for information about age, source of water supply, water treatment method, waste disposal practices, protection of water sources and health related problems. It was observed that the households involved use the borehole water for cooking, washing and bathing. Similarly, majority of the households also use the water for drinking. Two of the households not drinking theirs have neighbours who rely solely on them as source of drinking water. The health risk indicators, such as average daily dose (ADD) and hazard quotient (HQ) were calculated respectively using equations 2 and 3 [2] [15] [30]-[33] as shown below:

$$
\mathrm{ADD}=\frac{(\mathrm{CW} \times \mathrm{IR} \times \mathrm{EF} \times \mathrm{ED})}{(\mathrm{BW} \times \mathrm{AT})}
$$


Table 1. Physico-chemical parameters values in the borehole water samples.

\begin{tabular}{|c|c|c|c|c|c|c|c|c|c|}
\hline Location & $\mathrm{pH}$ & $\begin{array}{c}\text { Turbidity } \\
\text { (NTU) }\end{array}$ & $\begin{array}{l}\text { Dissolved } \\
\text { Oxygen } \\
(\mathrm{mg} / \mathrm{L})\end{array}$ & $\begin{array}{c}\text { Electrical } \\
\text { conductivity } \\
(\mathrm{mS} / \mathrm{cm})\end{array}$ & $\begin{array}{l}\text { Alkalinity } \\
(\mathrm{mg} / \mathrm{L})\end{array}$ & $\begin{array}{c}\text { Total } \\
\text { hardness } \\
(\mathrm{mg} / \mathrm{L})\end{array}$ & $\begin{array}{c}\text { Chloride } \\
(\mathrm{mg} / \mathrm{L})\end{array}$ & $\begin{array}{l}\text { Nitrate } \\
(\mathrm{mg} / \mathrm{L})\end{array}$ & $\begin{array}{c}\text { Sulphate } \\
\text { (mg/L) }\end{array}$ \\
\hline Erunwen 1 & $\begin{array}{c}5.28 \pm \\
0.38\end{array}$ & $\begin{array}{c}1.91 \pm \\
1.70\end{array}$ & $\begin{array}{c}6.42 \pm \\
0.98\end{array}$ & $\begin{array}{c}0.13 \pm \\
0.04\end{array}$ & $\begin{array}{c}18.30 \pm \\
6.91\end{array}$ & $\begin{array}{c}18.00 \pm \\
15.28\end{array}$ & $\begin{array}{c}8.50 \pm \\
2.60\end{array}$ & $\begin{array}{c}6.05 \pm \\
5.30\end{array}$ & $\begin{array}{c}5.17 \pm \\
4.84\end{array}$ \\
\hline Erunwen 2 & $\begin{array}{c}5.74 \pm \\
0.16\end{array}$ & $\begin{array}{c}2.03 \pm \\
3.58\end{array}$ & $\begin{array}{c}6.00 \pm \\
0.88\end{array}$ & $\begin{array}{c}0.65 \pm \\
0.90\end{array}$ & $\begin{array}{c}28.20 \pm \\
4.95\end{array}$ & $\begin{array}{c}14.67 \pm \\
2.49\end{array}$ & $\begin{array}{c}5.60 \pm \\
1.46\end{array}$ & $\begin{array}{c}3.93 \pm \\
2.15\end{array}$ & $\begin{array}{c}4.67 \pm \\
4.17\end{array}$ \\
\hline Erunwen 3 & $\begin{array}{c}5.49 \pm \\
0.54\end{array}$ & $\begin{array}{c}0.64 \pm \\
0.34\end{array}$ & $\begin{array}{c}5.95 \pm \\
0.76\end{array}$ & $\begin{array}{c}0.11 \pm \\
0.05\end{array}$ & $\begin{array}{c}18.60 \pm \\
5.18\end{array}$ & $\begin{array}{c}19.33 \pm \\
10.27\end{array}$ & $\begin{array}{c}4.00 \pm \\
2.17\end{array}$ & $\begin{array}{c}4.58 \pm \\
2.31\end{array}$ & $\begin{array}{c}3.33 \pm \\
1.89\end{array}$ \\
\hline Agbele & $\begin{array}{c}5.23 \pm \\
0.50\end{array}$ & $\begin{array}{c}0.86 \pm \\
0.37\end{array}$ & $\begin{array}{c}6.24 \pm \\
0.75\end{array}$ & $\begin{array}{c}0.15 \pm \\
0.06\end{array}$ & $\begin{array}{c}24.60 \pm \\
11.19\end{array}$ & $\begin{array}{c}25.67 \pm \\
28.52\end{array}$ & $\begin{array}{c}8.20 \pm \\
5.11\end{array}$ & $\begin{array}{c}4.79 \pm \\
2.73\end{array}$ & $\begin{array}{c}4.90 \pm \\
2.21\end{array}$ \\
\hline Kokoro Abu & $\begin{array}{c}4.23 \pm \\
0.31\end{array}$ & $\begin{array}{c}0.50 \pm \\
0.41\end{array}$ & $\begin{array}{c}5.94 \pm \\
1.12\end{array}$ & $\begin{array}{c}1.49 \pm \\
0.26\end{array}$ & $\begin{array}{c}15.88 \pm \\
4.92\end{array}$ & $\begin{array}{c}65.50 \pm \\
17.97\end{array}$ & $\begin{array}{c}69.60 \pm \\
1.36\end{array}$ & $\begin{array}{c}50.83 \pm \\
11.46\end{array}$ & $\begin{array}{c}5.00 \pm \\
3.89\end{array}$ \\
\hline Eyita 1 & $\begin{array}{c}6.01 \pm \\
0.22\end{array}$ & $\begin{array}{c}0.59 \pm \\
0.49\end{array}$ & $\begin{array}{c}6.21 \pm \\
0.66\end{array}$ & $\begin{array}{c}0.36 \pm \\
0.15\end{array}$ & $\begin{array}{c}34.40 \pm \\
14.18\end{array}$ & $\begin{array}{c}48.75 \pm \\
18.61\end{array}$ & $\begin{array}{c}8.30 \pm \\
1.33\end{array}$ & $\begin{array}{c}7.99 \pm \\
1.42\end{array}$ & $\begin{array}{c}2.00 \pm \\
0.71\end{array}$ \\
\hline Eyita 2 & $\begin{array}{c}5.68 \pm \\
0.69\end{array}$ & $\begin{array}{c}0.28 \pm \\
0.13\end{array}$ & $\begin{array}{c}6.28 \pm \\
0.55\end{array}$ & $\begin{array}{c}0.19 \pm \\
0.07\end{array}$ & $\begin{array}{c}19.30 \pm \\
9.82\end{array}$ & $\begin{array}{c}22.50 \pm \\
13.35\end{array}$ & $\begin{array}{c}8.00 \pm \\
3.78\end{array}$ & $\begin{array}{c}6.85 \pm \\
1.84\end{array}$ & $\begin{array}{c}3.33 \pm \\
1.03\end{array}$ \\
\hline Eyita 3 & $\begin{array}{c}6.07 \pm \\
0.27\end{array}$ & $\begin{array}{c}0.64 \pm \\
0.62\end{array}$ & $\begin{array}{c}6.40 \pm \\
0.71\end{array}$ & $\begin{array}{c}0.28 \pm \\
0.08\end{array}$ & $\begin{array}{c}34.60 \pm \\
10.80\end{array}$ & $\begin{array}{c}46.25 \pm \\
34.66\end{array}$ & $\begin{array}{c}6.50 \pm \\
2.19\end{array}$ & $\begin{array}{c}5.39 \pm \\
1.39\end{array}$ & $\begin{array}{c}2.00 \pm \\
0.41\end{array}$ \\
\hline Ikosi Ketu & $\begin{array}{c}4.22 \pm \\
0.43\end{array}$ & $\begin{array}{c}2.20 \pm \\
2.55\end{array}$ & $\begin{array}{c}5.58 \pm \\
0.67\end{array}$ & $\begin{array}{c}1.10 \pm \\
0.32\end{array}$ & $\begin{array}{c}23.00 \pm \\
12.39\end{array}$ & $\begin{array}{c}58.50 \pm \\
18.45\end{array}$ & $\begin{array}{c}40.60 \pm \\
3.81\end{array}$ & $\begin{array}{c}35.86 \pm \\
4.38\end{array}$ & $\begin{array}{c}2.00 \pm \\
0.41\end{array}$ \\
\hline Oshodi & $\begin{array}{c}4.75 \pm \\
0.28\end{array}$ & $\begin{array}{c}0.41 \pm \\
0.29\end{array}$ & $\begin{array}{c}5.59 \pm \\
0.47\end{array}$ & $\begin{array}{c}0.66 \pm \\
0.25\end{array}$ & $\begin{array}{c}24.30 \pm \\
8.39\end{array}$ & $\begin{array}{c}30.00 \pm \\
26.84\end{array}$ & $\begin{array}{c}35.90 \pm \\
11.92\end{array}$ & $\begin{array}{c}10.53 \pm \\
1.47\end{array}$ & $\begin{array}{c}2.50 \pm \\
0.71\end{array}$ \\
\hline Ogudu & $\begin{array}{c}4.17 \pm \\
0.26\end{array}$ & $\begin{array}{c}1.05 \pm \\
0.94\end{array}$ & $\begin{array}{c}5.39 \pm \\
0.94\end{array}$ & $\begin{array}{c}2.51 \pm \\
0.69\end{array}$ & $\begin{array}{c}17.80 \pm \\
4.57\end{array}$ & $\begin{array}{c}95.50 \pm \\
16.35\end{array}$ & $\begin{array}{c}72.50 \pm \\
47.22\end{array}$ & $\begin{array}{c}73.18 \pm \\
24.31\end{array}$ & $\begin{array}{c}12.67 \pm \\
8.72\end{array}$ \\
\hline Akesan 1 & $\begin{array}{c}5.09 \pm \\
0.29\end{array}$ & $\begin{array}{c}0.16 \pm \\
0.08\end{array}$ & $\begin{array}{c}6.87 \pm \\
1.87\end{array}$ & $\begin{array}{c}0.47 \pm \\
0.09\end{array}$ & $\begin{array}{c}20.50 \pm \\
7.81\end{array}$ & $\begin{array}{c}25.00 \pm \\
18.55\end{array}$ & $\begin{array}{c}12.60 \pm \\
3.34\end{array}$ & $\begin{array}{c}18.69 \pm \\
15.20\end{array}$ & $\begin{array}{c}4.50 \pm \\
2.86\end{array}$ \\
\hline Akesan 2 & $\begin{array}{c}5.59 \pm \\
0.22\end{array}$ & $\begin{array}{c}0.42 \pm \\
0.42\end{array}$ & $\begin{array}{c}6.33 \pm \\
1.70\end{array}$ & $\begin{array}{c}0.34 \pm \\
0.09\end{array}$ & $\begin{array}{c}27.00 \pm \\
6.00\end{array}$ & $\begin{array}{c}27.50 \pm \\
5.17\end{array}$ & $\begin{array}{c}8.50 \pm \\
2.59\end{array}$ & $\begin{array}{c}8.54 \pm \\
2.52\end{array}$ & $\begin{array}{c}5.67 \pm \\
2.05\end{array}$ \\
\hline Ilado & $\begin{array}{c}5.12 \pm \\
0.41\end{array}$ & $\begin{array}{c}0.39 \pm \\
0.08\end{array}$ & $\begin{array}{c}6.86 \pm \\
1.27\end{array}$ & $\begin{array}{c}0.13 \pm \\
0.09\end{array}$ & $\begin{array}{c}22.00 \pm \\
8.86\end{array}$ & $\begin{array}{c}19.00 \pm \\
10.61\end{array}$ & $\begin{array}{c}7.70 \pm \\
1.69\end{array}$ & $\begin{array}{c}3.95 \pm \\
2.93\end{array}$ & $\begin{array}{c}2.17 \pm \\
0.85\end{array}$ \\
\hline Ogijo $^{*}$ & $\begin{array}{c}5.70 \pm \\
0.27\end{array}$ & $\begin{array}{c}1.44 \pm \\
1.92\end{array}$ & $\begin{array}{c}6.12 \pm \\
0.91\end{array}$ & $\begin{array}{c}0.16 \pm \\
0.07\end{array}$ & $\begin{array}{c}25.70 \pm \\
9.56\end{array}$ & $\begin{array}{c}36.50 \pm \\
23.51\end{array}$ & $\begin{array}{c}8.20 \pm \\
2.48\end{array}$ & $\begin{array}{c}4.49 \pm \\
1.51\end{array}$ & $\begin{array}{c}2.83 \pm \\
0.24\end{array}$ \\
\hline Ibafo $^{*}$ & $\begin{array}{c}5.64 \pm \\
0.31\end{array}$ & $\begin{array}{c}0.26 \pm \\
0.10\end{array}$ & $\begin{array}{c}6.55 \pm \\
1.30\end{array}$ & $\begin{array}{c}0.74 \pm \\
0.16\end{array}$ & $\begin{array}{c}25.00 \pm \\
5.70\end{array}$ & $\begin{array}{c}57.50 \pm \\
12.44\end{array}$ & $\begin{array}{c}20.80 \pm \\
2.04\end{array}$ & $\begin{array}{c}24.43 \pm \\
7.74\end{array}$ & $\begin{array}{c}2.33 \pm \\
0.94\end{array}$ \\
\hline Magboro* & $\begin{array}{c}6.20 \pm \\
0.16\end{array}$ & $\begin{array}{c}0.28 \pm \\
0.13\end{array}$ & $\begin{array}{c}6.43 \pm \\
1.63\end{array}$ & $\begin{array}{c}0.39 \pm \\
0.07\end{array}$ & $\begin{array}{c}42.00 \pm \\
12.08\end{array}$ & $\begin{array}{c}35.70 \pm \\
5.45\end{array}$ & $\begin{array}{c}7.10 \pm \\
4.00\end{array}$ & $\begin{array}{c}6.76 \pm \\
1.96\end{array}$ & $\begin{array}{c}1.67 \pm \\
0.47\end{array}$ \\
\hline Ilupeju Agbara ${ }^{*}$ & $\begin{array}{c}4.68 \pm \\
0.40\end{array}$ & $\begin{array}{c}0.23 \pm \\
0.10\end{array}$ & $\begin{array}{c}6.44 \pm \\
1.43\end{array}$ & $\begin{array}{c}0.33 \pm \\
0.11\end{array}$ & $\begin{array}{c}14.50 \pm \\
6.78\end{array}$ & $\begin{array}{c}14.00 \pm \\
10.61\end{array}$ & $\begin{array}{c}11.80 \pm \\
3.41\end{array}$ & $\begin{array}{c}8.93 \pm \\
2.75\end{array}$ & $\begin{array}{c}3.67 \pm \\
1.25\end{array}$ \\
\hline Lusada $1^{*}$ & $\begin{array}{c}5.23 \pm \\
0.29\end{array}$ & $\begin{array}{c}0.24 \pm \\
0.08\end{array}$ & $\begin{array}{c}6.70 \pm \\
1.30\end{array}$ & $\begin{array}{c}0.16 \pm \\
0.07\end{array}$ & $\begin{array}{c}21.50 \pm \\
7.52\end{array}$ & $\begin{array}{c}21.50 \pm \\
10.16\end{array}$ & $\begin{array}{c}8.40 \pm \\
3.44\end{array}$ & $\begin{array}{c}4.36 \pm \\
2.86\end{array}$ & $\begin{array}{c}2.83 \pm \\
1.03\end{array}$ \\
\hline Lusada $2^{*}$ & $\begin{array}{c}5.60 \pm \\
0.31\end{array}$ & $\begin{array}{c}0.30 \pm \\
0.15\end{array}$ & $\begin{array}{c}6.94 \pm \\
1.46\end{array}$ & $\begin{array}{c}0.19 \pm \\
0.07\end{array}$ & $\begin{array}{c}22.50 \pm \\
8.37\end{array}$ & $\begin{array}{c}19.00 \pm \\
8.40\end{array}$ & $\begin{array}{c}7.59 \pm \\
2.59\end{array}$ & $\begin{array}{c}4.68 \pm \\
3.38\end{array}$ & $\begin{array}{c}6.17 \pm \\
6.25\end{array}$ \\
\hline WHO, 2011 & $6.5-8.5$ & - & - & - & - & - & - & 50.0 & - \\
\hline
\end{tabular}

Notes: * Ogun samples others are from Lagos State; ND, not detected; WHO (World Health Organization). 
Table 2. Mean metal concentrations in the borehole water samples.

\begin{tabular}{|c|c|c|c|c|c|c|c|c|c|c|c|}
\hline \multirow{2}{*}{ Location } & \multicolumn{10}{|c|}{$\mathrm{mg} / \mathrm{L}$} & \multirow{2}{*}{ MPI } \\
\hline & Ag & $\mathrm{Fe}$ & $\mathrm{Mn}$ & $\mathrm{Pb}$ & $\mathrm{Ni}$ & $\mathrm{Mg}$ & $\mathrm{Cd}$ & $\mathrm{Zn}$ & $\mathrm{Na}$ & $\mathrm{K}$ & \\
\hline Erunwen 1 & $\begin{array}{c}0.02 \pm \\
0.04\end{array}$ & $\begin{array}{c}0.03 \pm \\
0.06\end{array}$ & $\begin{array}{c}0.003 \pm \\
0.002\end{array}$ & $\begin{array}{c}0.05 \pm \\
0.09\end{array}$ & $\begin{array}{c}0.007 \pm \\
0.01\end{array}$ & $\begin{array}{c}0.03 \pm \\
0.04\end{array}$ & $\begin{array}{c}0.012 \pm \\
0.02\end{array}$ & $\begin{array}{c}0.001 \pm \\
0.0003\end{array}$ & $\begin{array}{c}0.06 \pm \\
0.02\end{array}$ & $\begin{array}{c}0.03 \pm \\
0.04\end{array}$ & 0.01 \\
\hline Erunwen 2 & $\begin{array}{c}0.03 \pm \\
0.06\end{array}$ & $\begin{array}{c}0.05 \pm \\
0.10\end{array}$ & $\begin{array}{c}0.004 \pm \\
0.003\end{array}$ & $\begin{array}{c}0.01 \pm \\
0.01\end{array}$ & $\begin{array}{c}0.008 \pm \\
0.01\end{array}$ & $\begin{array}{c}0.04 \pm \\
0.05\end{array}$ & $\begin{array}{c}0.013 \pm \\
0.01\end{array}$ & $\begin{array}{c}0.014 \pm \\
0.01\end{array}$ & $\begin{array}{c}0.05 \pm \\
0.05\end{array}$ & $\begin{array}{c}0.01 \pm \\
0.01\end{array}$ & 0.02 \\
\hline Erunwen 3 & $\begin{array}{c}0.04 \pm \\
0.07\end{array}$ & $\begin{array}{c}0.06 \pm \\
0.12\end{array}$ & $\begin{array}{c}0.002 \pm \\
0.002\end{array}$ & $\begin{array}{c}0.01 \pm \\
0.01\end{array}$ & $\begin{array}{c}0.009 \pm \\
0.01\end{array}$ & $\begin{array}{c}0.02 \pm \\
0.04\end{array}$ & $\begin{array}{c}1.121 \pm \\
1.12\end{array}$ & $\begin{array}{c}0.001 \pm \\
0.001\end{array}$ & $\begin{array}{c}0.03 \pm \\
0.03\end{array}$ & $\begin{array}{c}0.01 \pm \\
0.01\end{array}$ & 0.02 \\
\hline Agbele & $\begin{array}{c}0.21 \pm \\
0.32\end{array}$ & $\begin{array}{c}0.12 \pm \\
0.20\end{array}$ & $\begin{array}{c}0.002 \pm \\
0.003\end{array}$ & $\begin{array}{c}0.05 \pm \\
0.08\end{array}$ & $\begin{array}{c}0.015 \pm \\
0.01\end{array}$ & $\begin{array}{c}0.01 \pm \\
0.02\end{array}$ & ND & $\begin{array}{c}0.003 \pm \\
0.004\end{array}$ & $\begin{array}{c}0.01 \pm \\
0.02\end{array}$ & $\begin{array}{c}0.01 \pm \\
0.01\end{array}$ & 0.03 \\
\hline Kokoro Abu & $\begin{array}{c}0.06 \pm \\
0.11\end{array}$ & $\begin{array}{c}0.07 \pm \\
0.11\end{array}$ & $\begin{array}{c}0.004 \pm \\
0.002\end{array}$ & $\begin{array}{c}0.01 \pm \\
0.01\end{array}$ & $\begin{array}{c}0.007 \pm \\
0.01\end{array}$ & $\begin{array}{c}0.18 \pm \\
0.18\end{array}$ & $\begin{array}{c}0.002 \pm \\
0.0002\end{array}$ & $\begin{array}{c}0.002 \pm \\
0.001\end{array}$ & $\begin{array}{c}0.63 \pm \\
0.46\end{array}$ & $\begin{array}{c}0.37 \pm \\
0.43\end{array}$ & 0.03 \\
\hline Eyita 1 & $\begin{array}{c}0.10 \pm \\
0.20\end{array}$ & $\begin{array}{c}0.12 \pm \\
0.20\end{array}$ & $\begin{array}{c}0.003 \pm \\
0.002\end{array}$ & ND & $\begin{array}{c}0.013 \pm \\
0.01\end{array}$ & $\begin{array}{c}0.05 \pm \\
0.07\end{array}$ & ND & $\begin{array}{c}0.001 \pm \\
0.001\end{array}$ & $\begin{array}{c}0.14 \pm \\
0.10\end{array}$ & $\begin{array}{c}0.01 \pm \\
0.01\end{array}$ & 0.04 \\
\hline Eyita 2 & $\begin{array}{c}0.07 \pm \\
0.13\end{array}$ & $\begin{array}{c}0.06 \pm \\
0.11\end{array}$ & $\begin{array}{c}0.002 \pm \\
0.002\end{array}$ & $\begin{array}{c}0.003 \pm \\
0.01\end{array}$ & $\begin{array}{c}0.010 \pm \\
0.01\end{array}$ & $\begin{array}{c}0.04 \pm \\
0.05\end{array}$ & $\begin{array}{c}0.0001 \pm \\
0.0001\end{array}$ & $\begin{array}{c}0.002 \pm \\
0.001\end{array}$ & $\begin{array}{c}0.11 \pm \\
0.12\end{array}$ & $\begin{array}{c}0.01 \pm \\
0.01\end{array}$ & 0.01 \\
\hline Eyita 3 & $\begin{array}{c}0.06 \pm \\
0.12\end{array}$ & $\begin{array}{c}0.01 \pm \\
0.10\end{array}$ & $\begin{array}{c}0.001 \pm \\
0.002\end{array}$ & $\begin{array}{c}0.002 \pm \\
0.002\end{array}$ & $\begin{array}{c}0.008 \pm \\
0.01\end{array}$ & $\begin{array}{c}0.07 \pm \\
0.10\end{array}$ & ND & $\begin{array}{c}0.002 \pm \\
0.001\end{array}$ & $\begin{array}{c}0.09 \pm \\
0.10\end{array}$ & $\begin{array}{c}0.01 \pm \\
0.01\end{array}$ & 0.02 \\
\hline Ikosi Ketu & $\begin{array}{c}0.07 \pm \\
0.13\end{array}$ & $\begin{array}{c}0.06 \pm \\
0.10\end{array}$ & $\begin{array}{c}0.002 \pm \\
0.003\end{array}$ & $\begin{array}{c}0.001 \pm \\
0.003\end{array}$ & $\begin{array}{c}0.007 \pm \\
0.01\end{array}$ & $\begin{array}{c}0.16 \pm \\
0.14\end{array}$ & ND & $\begin{array}{c}0.001 \pm \\
0.001\end{array}$ & $\begin{array}{c}0.50 \pm \\
0.41\end{array}$ & $\begin{array}{c}0.10 \pm \\
0.11\end{array}$ & 0.03 \\
\hline Oshodi & $\begin{array}{c}0.09 \pm \\
0.18\end{array}$ & $\begin{array}{c}0.06 \pm \\
0.12\end{array}$ & $\begin{array}{c}0.001 \pm \\
0.002\end{array}$ & $\begin{array}{c}0.001 \pm \\
0.01\end{array}$ & $\begin{array}{c}0.007 \pm \\
0.01\end{array}$ & $\begin{array}{c}0.04 \pm \\
0.05\end{array}$ & $\begin{array}{c}0.0004 \pm \\
0.0004\end{array}$ & $\begin{array}{c}0.001 \pm \\
0.001\end{array}$ & $\begin{array}{c}0.36 \pm \\
0.30\end{array}$ & $\begin{array}{c}0.01 \pm \\
0.01\end{array}$ & 0.01 \\
\hline Ogudu & $\begin{array}{c}0.09 \pm \\
0.17\end{array}$ & $\begin{array}{c}0.05 \pm \\
0.10\end{array}$ & $\begin{array}{c}0.002 \pm \\
0.002\end{array}$ & $\begin{array}{c}0.002 \pm \\
0.004\end{array}$ & $\begin{array}{c}0.006 \pm \\
0.01\end{array}$ & $\begin{array}{c}0.19 \pm \\
0.16\end{array}$ & $\begin{array}{c}0.0002 \pm \\
0.0002\end{array}$ & $\begin{array}{c}0.003 \pm \\
0.003\end{array}$ & $\begin{array}{c}0.52 \pm \\
0.56\end{array}$ & $\begin{array}{c}0.96 \pm \\
1.56\end{array}$ & 0.02 \\
\hline Akesan 1 & ND & $\begin{array}{c}0.07 \pm \\
0.14\end{array}$ & $\begin{array}{c}0.14 \pm \\
0.24\end{array}$ & $\begin{array}{c}0.35 \pm \\
0.70\end{array}$ & $\begin{array}{c}0.093 \pm \\
0.18\end{array}$ & $\begin{array}{c}0.04 \pm \\
0.05\end{array}$ & $\begin{array}{c}0.006 \pm \\
0.01\end{array}$ & $\begin{array}{c}0.082 \pm \\
0.14\end{array}$ & $\begin{array}{c}0.71 \pm \\
0.46\end{array}$ & $\begin{array}{c}0.05 \pm \\
0.09\end{array}$ & 0.11 \\
\hline Akesan 2 & $\begin{array}{c}0.01 \pm \\
0.02\end{array}$ & $\begin{array}{c}0.05 \pm \\
0.08\end{array}$ & $\begin{array}{c}0.09 \pm \\
0.15\end{array}$ & $\begin{array}{c}0.23 \pm \\
0.03\end{array}$ & $\begin{array}{c}0.060 \pm \\
0.11\end{array}$ & $\begin{array}{c}0.01 \pm \\
0.01\end{array}$ & $\begin{array}{c}0.0013 \pm \\
0.001\end{array}$ & $\begin{array}{c}0.001 \pm \\
0.001\end{array}$ & $\begin{array}{c}0.48 \pm \\
0.20\end{array}$ & $\begin{array}{c}0.01 \pm \\
0.01\end{array}$ & 0.02 \\
\hline Ilado & $\begin{array}{c}0.01 \pm \\
0.03\end{array}$ & $\begin{array}{c}0.01 \pm \\
0.01\end{array}$ & $\begin{array}{c}0.09 \pm \\
0.15\end{array}$ & $\begin{array}{c}0.01 \pm \\
0.02\end{array}$ & $\begin{array}{c}0.002 \pm \\
2.07\end{array}$ & $\begin{array}{c}0.03 \pm \\
0.03\end{array}$ & $\begin{array}{c}0.0006 \pm \\
0.001\end{array}$ & $\begin{array}{c}0.024 \pm \\
0.02\end{array}$ & $\begin{array}{c}0.63 \pm \\
0.78\end{array}$ & $\begin{array}{c}0.01 \pm \\
0.01\end{array}$ & 0.02 \\
\hline Ogijo $^{*}$ & $\begin{array}{c}0.12 \pm \\
0.23\end{array}$ & $\begin{array}{c}0.07 \pm \\
0.13\end{array}$ & $\begin{array}{c}0.002 \pm \\
0.002\end{array}$ & $\begin{array}{c}0.001 \pm \\
0.001\end{array}$ & $\begin{array}{c}0.007 \pm \\
0.01\end{array}$ & $\begin{array}{c}0.04 \pm \\
0.05\end{array}$ & $\begin{array}{c}0.0001 \pm \\
0.0001\end{array}$ & $\begin{array}{c}0.002 \pm \\
0.002\end{array}$ & $\begin{array}{c}0.13 \pm \\
0.16\end{array}$ & $\begin{array}{c}0.03 \pm \\
0.03\end{array}$ & 0.01 \\
\hline Ibafo $^{*}$ & $\begin{array}{c}0.11 \pm \\
0.19\end{array}$ & $\begin{array}{c}0.05 \pm \\
0.09\end{array}$ & $\begin{array}{c}0.004 \pm \\
0.003\end{array}$ & $\begin{array}{c}0.001 \pm \\
0.002\end{array}$ & $\begin{array}{c}0.006 \pm \\
0.01\end{array}$ & $\begin{array}{c}0.13 \pm \\
0.10\end{array}$ & $\begin{array}{c}0.0001 \pm \\
0.0001\end{array}$ & $\begin{array}{c}0.001 \pm \\
0.001\end{array}$ & $\begin{array}{c}0.74 \pm \\
0.80\end{array}$ & $\begin{array}{c}1.08 \pm \\
1.56\end{array}$ & 0.02 \\
\hline Magboro $^{*}$ & $\begin{array}{c}0.003 \pm \\
0.01\end{array}$ & $\begin{array}{c}0.02 \pm \\
0.04\end{array}$ & $\begin{array}{c}0.04 \pm \\
0.07\end{array}$ & $\begin{array}{c}0.01 \pm \\
0.01\end{array}$ & $\begin{array}{c}0.029 \pm \\
0.05\end{array}$ & $\begin{array}{c}0.06 \pm \\
0.06\end{array}$ & $\begin{array}{c}0.0003 \pm \\
0.0003\end{array}$ & $\begin{array}{c}0.005 \pm \\
0.01\end{array}$ & $\begin{array}{c}0.76 \pm \\
0.23\end{array}$ & $\begin{array}{c}0.10 \pm \\
0.09\end{array}$ & 0.02 \\
\hline Ilupeju Agbara* & ND & $\begin{array}{c}0.05 \pm \\
0.08\end{array}$ & $\begin{array}{c}0.10 \pm \\
0.13\end{array}$ & $\begin{array}{c}0.002 \pm \\
0.004\end{array}$ & $\begin{array}{c}0.037 \pm \\
0.07\end{array}$ & $\begin{array}{c}0.08 \pm \\
0.09\end{array}$ & ND & $\begin{array}{c}0.001 \pm \\
0.001\end{array}$ & $\begin{array}{c}0.59 \pm \\
0.22\end{array}$ & $\begin{array}{c}0.13 \pm \\
0.02\end{array}$ & 0.07 \\
\hline Lusada $1^{*}$ & ND & $\begin{array}{c}0.06 \pm \\
0.10\end{array}$ & $\begin{array}{c}0.11 \pm \\
0.20\end{array}$ & $\begin{array}{c}0.002 \pm \\
0.003\end{array}$ & $\begin{array}{c}0.059 \pm \\
0.11\end{array}$ & $\begin{array}{c}0.04 \pm \\
0.07\end{array}$ & ND & $\begin{array}{c}0.003 \pm \\
0.003\end{array}$ & $\begin{array}{c}0.18 \pm \\
0.07\end{array}$ & $\begin{array}{c}0.003 \pm \\
0.01\end{array}$ & 0.05 \\
\hline Lusada $2^{*}$ & ND & $\begin{array}{c}0.09 \pm \\
0.15\end{array}$ & $\begin{array}{c}0.14 \pm \\
0.23\end{array}$ & $\begin{array}{c}0.01 \pm \\
0.01\end{array}$ & $\begin{array}{c}0.090 \pm \\
0.18\end{array}$ & $\begin{array}{c}0.03 \pm \\
0.03\end{array}$ & $\begin{array}{c}0.0001 \pm \\
0.000\end{array}$ & $\begin{array}{c}0.001 \pm \\
0.001\end{array}$ & $\begin{array}{c}0.28 \pm \\
0.12\end{array}$ & $\begin{array}{c}0.01 \pm \\
0.01\end{array}$ & 0.03 \\
\hline GBV & - & $\begin{array}{c}0.0016- \\
24.20\end{array}$ & $\begin{array}{l}\text { ND - } \\
16.00\end{array}$ & $\begin{array}{l}\text { ND - } \\
0.70\end{array}$ & $\begin{array}{c}\mathrm{ND}- \\
0.24\end{array}$ & $\begin{array}{l}2.00- \\
70.00\end{array}$ & ND - 0.20 & $\begin{array}{c}0.015 \text { - } \\
2.499\end{array}$ & $\begin{array}{c}5.70- \\
599.00\end{array}$ & $\begin{array}{l}1.60- \\
15.00\end{array}$ & \\
\hline WHO & - & - & 0.40 & 0.01 & 0.07 & - & 0.03 & - & - & - & - \\
\hline
\end{tabular}

Notes: *Ogun samples others are from Lagos State; ND, not detected; WHO, World Health Organization GBV, Global Background Values. 
where, ADD is the average daily dose from ingestion of metal in drinking water (mg/kg/day); CW is the concentration of a particular heavy metal in groundwater $(\mathrm{mg} / \mathrm{L})$; IR is the water ingestion rate (L/day); EF is the exposure frequency (day/year); ED is the exposure duration (years); BW is the body weight (Kg); AT is the average time of life expectancy (days). This study used the standard amount of water intake of $2 \mathrm{~L} /$ day recommended by the WHO [12]; while EF of 365 days year ${ }^{-1}$ recommended by the USEPA [33] was used. The life expectancy at birth of Nigerian is 54 years [35], this translates to an (AT) of 19,710 days. The average body weight used is assumed to be $72 \mathrm{Kg}$ for adult and $32.7 \mathrm{Kg}$ for child. Exposure duration was captured from the administered questionnaire.

\subsection{Non-Carcinogenic Risk Assessment}

The potential non-carcinogenic toxicity was estimated through the non-cancer hazard quotient (non-cancer HQ) shown in Equation (3) [2] [33].

$$
\text { Non-cancer HQ }=\frac{\mathrm{ADD}}{\mathrm{Rfd}}
$$

where, ADD is the average daily dose from ingestion of metal in drinking water (mg/kg/day); RfD is the oral reference dose (mg/kg/day) of a particular metal. The values (mg/kg/day): $\mathrm{Mn}, \mathrm{Pb}, \mathrm{Ni}, \mathrm{Cd}, \mathrm{Zn}$ and $\mathrm{Ag}, 1.4 \mathrm{E}+01$, $3.6 \mathrm{E}+03,2 \mathrm{E}+02,5 \mathrm{E}+04,3 \mathrm{E}+01$ and $5 \mathrm{E}+03$ respectively. The results are presented in Table 3 .

\subsection{Carcinogenic Risk Assessment for Cadmium}

The concentration of $\mathrm{Cd}$ in the borehole water was used to assess the probability of cancer developing in individuals over their lifetime [33]-[35]. This was evaluated using Equation (4).

$$
\mathrm{CR}=1-\exp (-\mathrm{ADD} \times \mathrm{SF})
$$

where, CR is the cancer risk; ADD, is as described earlier; SF is the oral cancer slope factor of Cd (6.3 mg/kg/ day). The calculated carcinogenic risk (CR) contribution of cadmium in the sampling locations is presented in Figure 2.

\subsection{Classification System}

The classification system proposed by Kempster et al., 1997 [13] was used to classify the borehole water examined in this study [36].

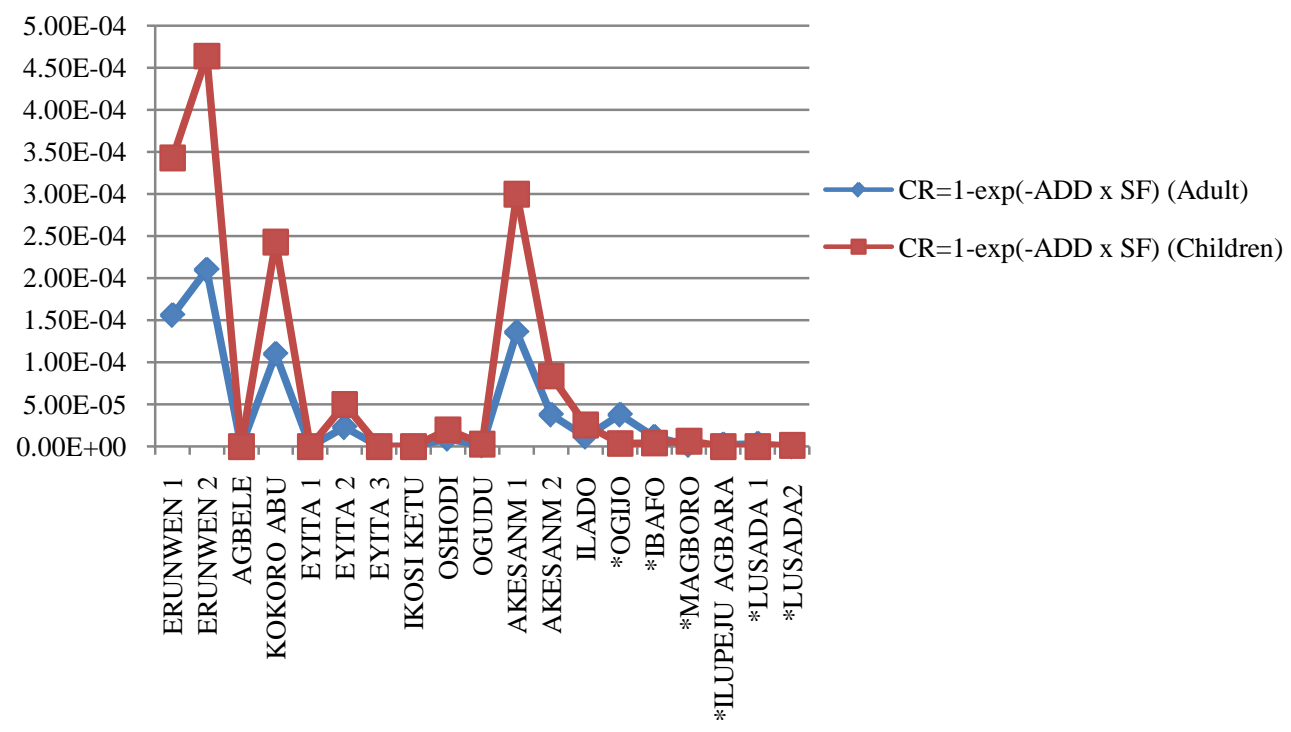

Figure 2. Carcinogen risk assessment values for cadmium in the water samples. Notes: $x$-axis, cancer risk quotient; y-axis, sampling locations. 
Table 3. Non cancer Health Quotient (HQ) for Lagos and Ogun states locations.

\begin{tabular}{|c|c|c|c|c|c|c|c|c|}
\hline Location & $\mathrm{Ag}$ & $\mathrm{Fe}$ & $\mathrm{Mn}$ & $\mathrm{Pb}$ & $\mathrm{Ni}$ & $\mathrm{Cd}$ & $\mathrm{Zn}$ & $\mathrm{HI}=\Sigma \mathrm{HQS}$ \\
\hline Erunwen 1 & $\begin{array}{c}8.23 \mathrm{E}-03 \\
(1.81 \mathrm{E}-02)\end{array}$ & $\begin{array}{c}2.06 \mathrm{E}-04 \\
(4.53 \mathrm{E}-04)\end{array}$ & $\begin{array}{c}4.41 \mathrm{E}-05 \\
(9.71 \mathrm{E}-05)\end{array}$ & $\begin{array}{c}2.94 \mathrm{E}-02 \\
(6.47 \mathrm{E}-02)\end{array}$ & $\begin{array}{c}7.20 \mathrm{E}-04 \\
(1.59 \mathrm{E}-03)\end{array}$ & $\begin{array}{c}4.94 \mathrm{E}-02 \\
(1.09 \mathrm{E}-01)\end{array}$ & $\begin{array}{c}6.86 \mathrm{E}-06 \\
(1.51 \mathrm{E}-05)\end{array}$ & $\begin{array}{c}8.80 \mathrm{E}-02 \\
(1.94 \mathrm{E}-01)\end{array}$ \\
\hline Erunwen 2 & $\begin{array}{c}1.54 \mathrm{E}-02 \\
(3.40 \mathrm{E}-02)\end{array}$ & $\begin{array}{c}4.29 \mathrm{E}-04 \\
(9.44 \mathrm{E}-04)\end{array}$ & $\begin{array}{c}7.35 \mathrm{E}-05 \\
(1.62 \mathrm{E}-04)\end{array}$ & $\begin{array}{c}7.35 \mathrm{E}-03 \\
(1.62 \mathrm{E}-02)\end{array}$ & $\begin{array}{c}1.03 \mathrm{E}-03 \\
(2.27 \mathrm{E}-03)\end{array}$ & $\begin{array}{c}6.69 \mathrm{E}-02 \\
(1.47 \mathrm{E}-01)\end{array}$ & $\begin{array}{c}1.20 \mathrm{E}-04 \\
(2.64 \mathrm{E}-04)\end{array}$ & $\begin{array}{c}9.13 \mathrm{E}-02 \\
(2.01 \mathrm{E}-01)\end{array}$ \\
\hline Erunwen 3 & $\begin{array}{c}2.88 \mathrm{E}-02 \\
(6.34 \mathrm{E}-02)\end{array}$ & $\begin{array}{c}7.20 \mathrm{E}-04 \\
(1.59 \mathrm{E}-03)\end{array}$ & $\begin{array}{c}5.14 \mathrm{E}-05 \\
(1.13 \mathrm{E}-04)\end{array}$ & $\begin{array}{c}1.03 \mathrm{E}-02 \\
(2.27 \mathrm{E}-02)\end{array}$ & $\begin{array}{c}1.62 \mathrm{E}-03 \\
(3.57 \mathrm{E}-03)\end{array}$ & $\begin{array}{c}8.07 \mathrm{E}+00 \\
(1.78 \mathrm{E}+01)\end{array}$ & $\begin{array}{c}1.20 \mathrm{E}-05 \\
(2.64 \mathrm{E}-05)\end{array}$ & $\begin{array}{c}8.11 \mathrm{E}+00 \\
(1.79 \mathrm{E}+01)\end{array}$ \\
\hline Agbele & $\begin{array}{c}1.08 \mathrm{E}-01 \\
(2.38 \mathrm{E}-01)\end{array}$ & $\begin{array}{c}1.03 \mathrm{E}-03 \\
(2.27 \mathrm{E}-03)\end{array}$ & $\begin{array}{c}3.67 \mathrm{E}-05 \\
(8.09 \mathrm{E}-05)\end{array}$ & $\begin{array}{c}3.67 \mathrm{E}-02 \\
(8.09 \mathrm{E}-02)\end{array}$ & $\begin{array}{c}1.93 \mathrm{E}-03 \\
(4.25 \mathrm{E}-03)\end{array}$ & $\begin{array}{c}0.00 \mathrm{E}+00 \\
(0.00 \mathrm{E}+00)\end{array}$ & $\begin{array}{c}2.57 \mathrm{E}-05 \\
(5.66 \mathrm{E}-05)\end{array}$ & $\begin{array}{c}1.48 \mathrm{E}-01 \\
(3.25 \mathrm{E}-01)\end{array}$ \\
\hline Kokoro Abu & $\begin{array}{c}1.05 \mathrm{E}-01 \\
(2.31 \mathrm{E}-01)\end{array}$ & $\begin{array}{c}2.04 \mathrm{E}-03 \\
(4.49 \mathrm{E}-03)\end{array}$ & $\begin{array}{c}2.50 \mathrm{E}-04 \\
(5.50 \mathrm{E}-04)\end{array}$ & $\begin{array}{c}2.50 \mathrm{E}-02 \\
(5.50 \mathrm{E}-02)\end{array}$ & $\begin{array}{c}3.06 \mathrm{E}-03 \\
(6.74 \mathrm{E}-03)\end{array}$ & $\begin{array}{c}3.50 \mathrm{E}-02 \\
(7.70 \mathrm{E}-02)\end{array}$ & $\begin{array}{c}5.83 \mathrm{E}-05 \\
(1.28 \mathrm{E}-04)\end{array}$ & $\begin{array}{c}1.70 \mathrm{E}-01 \\
(3.75 \mathrm{E}-01)\end{array}$ \\
\hline Eyita 1 & $\begin{array}{c}1.34 \mathrm{E}-01 \\
(2.94 \mathrm{E}-01)\end{array}$ & $\begin{array}{c}2.68 \mathrm{E}-03 \\
(5.89 \mathrm{E}-03)\end{array}$ & $\begin{array}{c}1.43 \mathrm{E}-04 \\
(3.15 \mathrm{E}-04)\end{array}$ & $\begin{array}{c}0.00 \mathrm{E}+00 \\
(0.00 \mathrm{E}+00)\end{array}$ & $\begin{array}{c}4.35 \mathrm{E}-03 \\
(9.57 \mathrm{E}-03)\end{array}$ & $\begin{array}{c}0.00 \mathrm{E}+00 \\
(0.00 \mathrm{E}+00)\end{array}$ & $\begin{array}{c}2.23 \mathrm{E}-05 \\
(4.91 \mathrm{E}-05)\end{array}$ & $\begin{array}{c}1.41 \mathrm{E}-01 \\
(3.10 \mathrm{E}-01)\end{array}$ \\
\hline Eyita 2 & $\begin{array}{c}5.04 \mathrm{E}-02 \\
(1.11 \mathrm{E}-01)\end{array}$ & $\begin{array}{c}7.20 \mathrm{E}-04 \\
(1.59 \mathrm{E}-03)\end{array}$ & $\begin{array}{c}5.14 \mathrm{E}-05 \\
(1.13 \mathrm{E}-04)\end{array}$ & $\begin{array}{c}3.09 \mathrm{E}-03 \\
(6.80 \mathrm{E}-03)\end{array}$ & $\begin{array}{c}1.80 \mathrm{E}-03 \\
(3.96 \mathrm{E}-03)\end{array}$ & $\begin{array}{c}7.20 \mathrm{E}-03 \\
(1.59 \mathrm{E}-02)\end{array}$ & $\begin{array}{c}2.40 \mathrm{E}-05 \\
(5.29 \mathrm{E}-05)\end{array}$ & $\begin{array}{c}6.33 \mathrm{E}-02 \\
(1.39 \mathrm{E}-01)\end{array}$ \\
\hline Eyita 3 & $\begin{array}{c}1.85 \mathrm{E}-02 \\
(4.08 \mathrm{E}-02)\end{array}$ & $\begin{array}{c}5.14 \mathrm{E}-05 \\
(1.13 \mathrm{E}-04)\end{array}$ & $\begin{array}{c}1.10 \mathrm{E}-05 \\
(2.43 \mathrm{E}-05)\end{array}$ & $\begin{array}{c}8.82 \mathrm{E}-04 \\
(1.94 \mathrm{E}-03)\end{array}$ & $\begin{array}{c}6.17 \mathrm{E}-04 \\
(1.36 \mathrm{E}-03)\end{array}$ & $\begin{array}{c}0.00 \mathrm{E}+00 \\
(0.00 \mathrm{E}+00)\end{array}$ & $\begin{array}{c}1.03 \mathrm{E}-05 \\
(2.27 \mathrm{E}-05)\end{array}$ & $\begin{array}{c}2.01 \mathrm{E}-02 \\
(4.42 \mathrm{E}-02)\end{array}$ \\
\hline Ikosi Ketu & $\begin{array}{c}7.20 \mathrm{E}-02 \\
(1.59 \mathrm{E}-01)\end{array}$ & $\begin{array}{c}1.03 \mathrm{E}-03 \\
(2.27 \mathrm{E}-03)\end{array}$ & $\begin{array}{c}7.35 \mathrm{E}-05 \\
(1.62 \mathrm{E}-04)\end{array}$ & $\begin{array}{c}1.47 \mathrm{E}-03 \\
(3.24 \mathrm{E}-03)\end{array}$ & $\begin{array}{c}1.80 \mathrm{E}-03 \\
(3.96 \mathrm{E}-03)\end{array}$ & $\begin{array}{c}0.00 \mathrm{E}+00 \\
(0.00 \mathrm{E}+00)\end{array}$ & $\begin{array}{c}1.71 \mathrm{E}-05 \\
(3.78 \mathrm{E}-05)\end{array}$ & $\begin{array}{c}7.64 \mathrm{E}-02 \\
(1.68 \mathrm{E}-01)\end{array}$ \\
\hline Oshodi & $\begin{array}{c}6.48 \mathrm{E}-02 \\
(1.43 \mathrm{E}-01)\end{array}$ & $\begin{array}{c}7.20 \mathrm{E}-04 \\
(1.59 \mathrm{E}-03)\end{array}$ & $\begin{array}{c}2.57 \mathrm{E}-05 \\
(5.66 \mathrm{E}-05)\end{array}$ & $\begin{array}{c}1.03 \mathrm{E}-03 \\
(2.27 \mathrm{E}-03)\end{array}$ & $\begin{array}{c}1.26 \mathrm{E}-03 \\
(2.78 \mathrm{E}-03)\end{array}$ & $\begin{array}{c}2.88 \mathrm{E}-03 \\
(6.34 \mathrm{E}-03)\end{array}$ & $\begin{array}{c}1.20 \mathrm{E}-05 \\
(2.64 \mathrm{E}-05)\end{array}$ & $\begin{array}{c}7.07 \mathrm{E}-02 \\
(1.56 \mathrm{E}-01)\end{array}$ \\
\hline Ogudu & $\begin{array}{c}1.85 \mathrm{E}-02 \\
(4.08 \mathrm{E}-02)\end{array}$ & $\begin{array}{c}1.71 \mathrm{E}-04 \\
(3.78 \mathrm{E}-04)\end{array}$ & $\begin{array}{c}1.47 \mathrm{E}-05 \\
(3.24 \mathrm{E}-05)\end{array}$ & $\begin{array}{c}5.88 \mathrm{E}-04 \\
(1.29 \mathrm{E}-03)\end{array}$ & $\begin{array}{c}3.09 \mathrm{E}-04 \\
(6.80 \mathrm{E}-04)\end{array}$ & $\begin{array}{c}4.12 \mathrm{E}-04 \\
(9.06 \mathrm{E}-04)\end{array}$ & $\begin{array}{c}1.03 \mathrm{E}-05 \\
(2.27 \mathrm{E}-05)\end{array}$ & $\begin{array}{c}2.00 \mathrm{E}-02 \\
(4.41 \mathrm{E}-02)\end{array}$ \\
\hline Akesan 1 & $\begin{array}{c}0.00 \mathrm{E}+00 \\
(0.00 \mathrm{E}+00)\end{array}$ & $\begin{array}{c}8.40 \mathrm{E}-04 \\
(1.85 \mathrm{E}-03)\end{array}$ & $\begin{array}{c}3.60 \mathrm{E}-03 \\
(7.93 \mathrm{E}-03)\end{array}$ & $\begin{array}{c}3.60 \mathrm{E}-01 \\
(7.93 \mathrm{E}-01)\end{array}$ & $\begin{array}{c}1.67 \mathrm{E}-02 \\
(3.69 \mathrm{E}-02)\end{array}$ & $\begin{array}{c}4.32 \mathrm{E}-02 \\
(9.51 \mathrm{E}-02)\end{array}$ & $\begin{array}{c}9.84 \mathrm{E}-04 \\
(2.17 \mathrm{E}-03)\end{array}$ & $\begin{array}{c}4.25 \mathrm{E}-01 \\
(9.37 \mathrm{E}-01)\end{array}$ \\
\hline Akesan 2 & $\begin{array}{c}9.26 \mathrm{E}-03 \\
(2.04 \mathrm{E}-02)\end{array}$ & $\begin{array}{c}7.72 \mathrm{E}-04 \\
(1.70 \mathrm{E}-03)\end{array}$ & $\begin{array}{c}2.98 \mathrm{E}-03 \\
(6.55 \mathrm{E}-03)\end{array}$ & $\begin{array}{c}3.04 \mathrm{E}-01 \\
(6.70 \mathrm{E}-01)\end{array}$ & $\begin{array}{c}1.39 \mathrm{E}-02 \\
(3.06 \mathrm{E}-02)\end{array}$ & $\begin{array}{c}1.20 \mathrm{E}-02 \\
(2.65 \mathrm{E}-02)\end{array}$ & $\begin{array}{c}1.54 \mathrm{E}-05 \\
(3.40 \mathrm{E}-05)\end{array}$ & $\begin{array}{c}3.43 \mathrm{E}-01 \\
(7.56 \mathrm{E}-01)\end{array}$ \\
\hline Ilado & $\begin{array}{c}6.17 \mathrm{E}-03 \\
(1.36 \mathrm{E}-02)\end{array}$ & $\begin{array}{c}1.03 \mathrm{E}-04 \\
(2.27 \mathrm{E}-04)\end{array}$ & $\begin{array}{c}1.98 \mathrm{E}-03 \\
(4.37 \mathrm{E}-03)\end{array}$ & $\begin{array}{c}8.82 \mathrm{E}-03 \\
(1.94 \mathrm{E}-02)\end{array}$ & $\begin{array}{c}3.09 \mathrm{E}-04 \\
(6.80 \mathrm{E}-04)\end{array}$ & $\begin{array}{c}3.70 \mathrm{E}-03 \\
(8.15 \mathrm{E}-03)\end{array}$ & $\begin{array}{c}2.47 \mathrm{E}-04 \\
(5.44 \mathrm{E}-04)\end{array}$ & $\begin{array}{c}2.13 \mathrm{E}-02 \\
(4.70 \mathrm{E}-02)\end{array}$ \\
\hline Ogijo ${ }^{*}$ & $\begin{array}{c}6.17 \mathrm{E}-02 \\
(1.36 \mathrm{E}-01)\end{array}$ & $\begin{array}{c}6.00 \mathrm{E}-04 \\
(1.32 \mathrm{E}-03)\end{array}$ & $\begin{array}{c}3.67 \mathrm{E}-05 \\
(8.09 \mathrm{E}-05)\end{array}$ & $\begin{array}{c}7.35 \mathrm{E}-04 \\
(1.62 \mathrm{E}-03)\end{array}$ & $\begin{array}{c}9.00 \mathrm{E}-04 \\
(1.98 \mathrm{E}-03)\end{array}$ & $\begin{array}{c}5.14 \mathrm{E}-04 \\
(1.13 \mathrm{E}-03)\end{array}$ & $\begin{array}{c}1.71 \mathrm{E}-05 \\
(3.78 \mathrm{E}-05)\end{array}$ & $\begin{array}{c}6.45 \mathrm{E}-02 \\
(1.42 \mathrm{E}-01)\end{array}$ \\
\hline Ibafo $^{*}$ & $\begin{array}{c}6.79 \mathrm{E}-02 \\
(1.49 \mathrm{E}-01)\end{array}$ & $\begin{array}{c}5.14 \mathrm{E}-04 \\
(1.13 \mathrm{E}-03)\end{array}$ & $\begin{array}{c}8.82 \mathrm{E}-05 \\
(1.94 \mathrm{E}-04)\end{array}$ & $\begin{array}{c}8.82 \mathrm{E}-04 \\
(1.94 \mathrm{E}-03)\end{array}$ & $\begin{array}{c}9.26 \mathrm{E}-04 \\
(2.04 \mathrm{E}-03)\end{array}$ & $\begin{array}{c}6.17 \mathrm{E}-04 \\
(1.36 \mathrm{E}-03)\end{array}$ & $\begin{array}{c}1.03 \mathrm{E}-05 \\
(2.27 \mathrm{E}-05)\end{array}$ & $\begin{array}{c}7.09 \mathrm{E}-02 \\
(1.56 \mathrm{E}-01)\end{array}$ \\
\hline Magboro* & $\begin{array}{c}9.26 \mathrm{E}-04 \\
(2.04 \mathrm{E}-03)\end{array}$ & $\begin{array}{c}1.03 \mathrm{E}-04 \\
(2.27 \mathrm{E}-04)\end{array}$ & $\begin{array}{c}4.41 \mathrm{E}-04 \\
(9.71 \mathrm{E}-04)\end{array}$ & $\begin{array}{c}4.41 \mathrm{E}-03 \\
(9.71 \mathrm{E}-03)\end{array}$ & $\begin{array}{c}2.24 \mathrm{E}-03 \\
(4.93 \mathrm{E}-03)\end{array}$ & $\begin{array}{c}9.26 \mathrm{E}-04 \\
(2.04 \mathrm{E}-03)\end{array}$ & $\begin{array}{c}2.57 \mathrm{E}-05 \\
(5.66 \mathrm{E}-05)\end{array}$ & $\begin{array}{c}9.07 \mathrm{E}-03 \\
(2.00 \mathrm{E}-02)\end{array}$ \\
\hline $\begin{array}{l}\text { Ilupeju } \\
\text { Agbara* }\end{array}$ & $\begin{array}{c}0.00 \mathrm{E}+00 \\
(0.00 \mathrm{E}+00)\end{array}$ & $\begin{array}{c}4.29 \mathrm{E}-04 \\
(9.44 \mathrm{E}-04)\end{array}$ & $\begin{array}{c}1.84 \mathrm{E}-03 \\
(4.05 \mathrm{E}-03)\end{array}$ & $\begin{array}{c}1.47 \mathrm{E}-03 \\
(3.24 \mathrm{E}-03)\end{array}$ & $\begin{array}{c}4.76 \mathrm{E}-03 \\
(1.05 \mathrm{E}-02)\end{array}$ & $\begin{array}{c}0.00 \mathrm{E}+00 \\
(0.00 \mathrm{E}+00)\end{array}$ & $\begin{array}{c}8.57 \mathrm{E}-06 \\
(1.89 \mathrm{E}-05)\end{array}$ & $\begin{array}{c}8.50 \mathrm{E}-03 \\
(1.87 \mathrm{E}-02)\end{array}$ \\
\hline Lusada $1^{*}$ & $\begin{array}{c}0.00 E+00 \\
(0.00 E+00)\end{array}$ & $\begin{array}{c}3.09 \mathrm{E}-04 \\
(6.80 \mathrm{E}-04)\end{array}$ & $\begin{array}{c}1.21 \mathrm{E}-03 \\
(2.67 \mathrm{E}-03)\end{array}$ & $\begin{array}{c}8.82 \mathrm{E}-04 \\
(1.94 \mathrm{E}-03)\end{array}$ & $\begin{array}{c}4.55 \mathrm{E}-03 \\
(1.00 \mathrm{E}-02)\end{array}$ & $\begin{array}{c}0.00 \mathrm{E}+00 \\
(0.00 \mathrm{E}+00)\end{array}$ & $\begin{array}{c}1.54 \mathrm{E}-05 \\
(3.40 \mathrm{E}-05)\end{array}$ & $\begin{array}{c}6.97 \mathrm{E}-03 \\
(1.53 \mathrm{E}-02)\end{array}$ \\
\hline Lusada $2^{*}$ & $\begin{array}{c}0.00 \mathrm{E}+00 \\
(0.00 \mathrm{E}+00)\end{array}$ & $\begin{array}{c}3.09 \mathrm{E}-04 \\
(6.80 \mathrm{E}-04)\end{array}$ & $\begin{array}{c}1.03 \mathrm{E}-03 \\
(2.27 \mathrm{E}-03)\end{array}$ & $\begin{array}{c}2.94 \mathrm{E}-03 \\
(6.47 \mathrm{E}-03)\end{array}$ & $\begin{array}{c}4.63 \mathrm{E}-03 \\
(1.02 \mathrm{E}-02)\end{array}$ & $\begin{array}{c}2.06 \mathrm{E}-04 \\
(4.53 \mathrm{E}-04)\end{array}$ & $\begin{array}{c}3.43 \mathrm{E}-06 \\
(7.55 \mathrm{E}-06)\end{array}$ & $\begin{array}{c}9.12 \mathrm{E}-03 \\
(2.01 \mathrm{E}-02)\end{array}$ \\
\hline
\end{tabular}

Notes: The values in parentheses are for the children; E, exponential. Continued on next page. ${ }^{*}$ Ogun State samples. 
Class 0: "This is defined as the ideal water quality suitable for lifetime use, with no adverse effects on the user".

Class I: "Water in this Class is safe for lifetime use, but falls short of the ideal water quality in that there may be instances of adverse health effects, but these are usually mild health effects, almost sub-clinical and difficult to demonstrate. Aesthetic effects may however be apparent".

Class II: "Water in this Class is defined as that where adverse health effects may become more common particularly with prolonged use over many years, or with lifetime use. This class represents water suitable for shortterm or emergency use only, but not necessarily suitable for continuous use over a lifetime”.

Class III: "This water has constituents in concentration range where serious health effects might be anticipated particularly in infants or elderly people with short-term use, and even more so with longer term use. The water in this class is not suitable for use as drinking water without adequate treatment to shift the water into a lower and safer Class”. The classification of water from Lagos-Ogun axis is presented in Table 4.

\subsection{Statistical Analyses}

Pearson's correlation coefficient and t-test statistical analyses were used at 95\% confidence level to evaluate results obtained from the physic-chemical analyses of the water samples from Lagos and Ogun States. The $\mathrm{r}$ values are indicated in Table 5 and Table 6 respectively.

\section{Results and Discussion}

The $\mathrm{pH}$ values ranged from 4.17 to 6.07 and 4.68 to 6.20 in Lagos and Ogun borehole drinking water samples (Table 1) respectively and were generally below the permissible limit (6.50 - 8.50) set by World Health Organization [38]. Similarly, they vary from values reported earlier for Chikhwawa, Malawi borehole water (6.90 7.70), Lao PDR (3.53 - 7.92), Swat, Northern Pakistan (6.50 - 8.60) respectively [2] [10] [15]. This may be an indication of the unwholesomeness of the water for drinking. The $\mathrm{pH}$ of drinking water has no immediate direct effects on human health but acidic water has some indirect health effects by bringing changes in other water quality parameters such as solubility of metals [16]. In sensitive individuals gastrointestinal irritation may also occur [13]. In the present study, the EC $(\mathrm{mS} / \mathrm{cm})$ were found to be between 0.11 and 2.51 (Table 1), whereas, the Malawi's and Lao PDR values are respectively, 220.70 - 6574.30 and 20.70 - 2840.0. Nevertheless, turbidity (NTU) of the Lagos-Ogun axis borehole water of 0.23 - 2.20 were found to be generally higher than Chikhwawa, Malawi values (ND - 3.90).

Characteristically, anions also play important roles in the quality of drinking water. The Malawi's chloride, nitrate and sulphate (mg/L) levels (8.10 - 426.0, ND - 177.60, 4.50 - 294.40 respectively), were generally higher

Table 4. Parameters, values and classification of drinking water quality proposed by Kempster et al. 1997.

\begin{tabular}{|c|c|c|c|c|}
\hline Constituent & Class 0 & Class I & Class II & Class III \\
\hline Electrical conductivity (mS/cm) & $0-0.7$ & $0.7-1.50$ & $1.50-3.70$ & $>3.70$ \\
\hline $\mathrm{pH}$ & $6.0-9.0$ & $5-6$ or $9-9.5$ & $4-5$ or $9.5-10$ & $<4$ or $>10$ \\
\hline Nitrate (mg/L) & $0-6$ & $6-10$ & $10-20$ & 20 \\
\hline Sulphate (mg/L) & $0-200$ & $200-400$ & $400-600$ & $>600$ \\
\hline Chloride (mg/L) & $0-100$ & $100-200$ & $200-600$ & $>600$ \\
\hline Iron (mg/L) & $0-0.1$ & $0.1-0.2$ & $0.2-2.0$ & $>2.0$ \\
\hline Manganese (mg/L) & $0-0.05$ & $0.05-0.1$ & $0.1-1.0$ & $>1.0$ \\
\hline Cadmium (mg/L) & $0-0.005$ & $0.005-0.01$ & $0.01-0.02$ & $>0.02$ \\
\hline Zinc (mg/L) & $0-3.0$ & $3.0-5.0$ & $5.0-10.0$ & $>10.0$ \\
\hline Magnesium (mg/L) & $0-30$ & $30-70$ & $70-100$ & $>100$ \\
\hline Sodium (mg/L) & $0-100$ & $100-200$ & $200-400$ & $>400$ \\
\hline
\end{tabular}


Table 5. Pearson correlation coefficient (r) of individual Physico chemical parameters in Lagos and Ogun water samples.

\begin{tabular}{|c|c|c|c|c|c|c|c|c|c|}
\hline & $\mathrm{pH}$ & Turbidity & $\begin{array}{l}\text { Dissolved } \\
\text { Oxygen }\end{array}$ & $\begin{array}{c}\text { Electrical } \\
\text { Conductivity }\end{array}$ & $\begin{array}{c}\text { Total } \\
\text { alkalinity }\end{array}$ & Chloride & Nitrate & Sulphate & $\begin{array}{c}\text { Total } \\
\text { hardness }\end{array}$ \\
\hline $\mathrm{pH}$ & $\begin{array}{c}1.000 \\
(1.000)\end{array}$ & $\begin{array}{l}-0.19 \\
(0.22)\end{array}$ & $\begin{array}{c}0.53 \\
(-0.11)\end{array}$ & $\begin{array}{l}-0.75 \\
(0.17)\end{array}$ & $\begin{array}{c}0.66 \\
(0.91)\end{array}$ & $\begin{array}{l}-0.87 \\
(-0.16)\end{array}$ & $\begin{array}{l}-0.81 \\
(0.03)\end{array}$ & $\begin{array}{c}-0.43 \\
(-0.31)\end{array}$ & $\begin{array}{l}-0.59 \\
(0.57)\end{array}$ \\
\hline Turbidity & & $\begin{array}{l}1.000 \\
(1.000)\end{array}$ & $\begin{array}{c}-0.38 \\
(-0.71)\end{array}$ & $\begin{array}{c}0.19 \\
(-0.37)\end{array}$ & $\begin{array}{c}0.00 \\
(0.06)\end{array}$ & $\begin{array}{l}-0.67 \\
(-0.02)\end{array}$ & $\begin{array}{c}0.12 \\
(-0.29)\end{array}$ & $\begin{array}{c}0.11 \\
(-0.11)\end{array}$ & $\begin{array}{c}0.07 \\
(0.19)\end{array}$ \\
\hline $\begin{array}{l}\text { Dissolved } \\
\text { Oxygen }\end{array}$ & & & $\begin{array}{l}1.000 \\
(1.000)\end{array}$ & $\begin{array}{l}-0.68 \\
(-0.05)\end{array}$ & $\begin{array}{c}0.15 \\
(-0.21)\end{array}$ & $\begin{array}{c}-0.67 \\
(-0.02)\end{array}$ & $\begin{array}{l}-0.58 \\
(-0.02)\end{array}$ & $\begin{array}{l}-0.36 \\
(0.64)\end{array}$ & $\begin{array}{l}-0.60 \\
(-0.33)\end{array}$ \\
\hline $\begin{array}{l}\text { Electrical } \\
\text { Conductivity }\end{array}$ & & & & $\begin{array}{c}1.000 \\
(1.000)\end{array}$ & $\begin{array}{l}-0.34 \\
(0.18)\end{array}$ & $\begin{array}{c}0.93 \\
(0.88)\end{array}$ & $\begin{array}{c}0.96 \\
(0.96)\end{array}$ & $\begin{array}{c}0.72 \\
(-0.42)\end{array}$ & $\begin{array}{c}0.88 \\
(0.76)\end{array}$ \\
\hline $\begin{array}{c}\text { Total } \\
\text { Alkalinity }\end{array}$ & & & & & $\begin{array}{l}1.000 \\
(1.000)\end{array}$ & $\begin{array}{l}-0.46 \\
(-0.25)\end{array}$ & $\begin{array}{l}-0.46 \\
(-0.04)\end{array}$ & $\begin{array}{c}-0.43 \\
(-0.52)\end{array}$ & $\begin{array}{l}-0.10 \\
(0.45)\end{array}$ \\
\hline Chloride & & & & & & $\begin{array}{c}1.000 \\
(1.000)\end{array}$ & $\begin{array}{c}0.94 \\
(0.98)\end{array}$ & $\begin{array}{c}0.55 \\
(-0.25)\end{array}$ & $\begin{array}{c}0.85 \\
(0.66)\end{array}$ \\
\hline Nitrate & & & & & & & $\begin{array}{c}1.000 \\
(1.000)\end{array}$ & $\begin{array}{c}0.69 \\
(-0.32)\end{array}$ & $\begin{array}{c}0.90 \\
(0.76)\end{array}$ \\
\hline Sulphate & & & & & & & & $\begin{array}{c}1.000 \\
(1.000)\end{array}$ & $\begin{array}{c}0.53 \\
(-0.57)\end{array}$ \\
\hline $\begin{array}{c}\text { Total } \\
\text { Hardness }\end{array}$ & & & & & & & & & $\begin{array}{c}1.000 \\
(1.000)\end{array}$ \\
\hline
\end{tabular}

Note: Values in parentheses are for Ogun samples.

Table 6. Pearson correlation coefficient (r) of individual metals in Lagos and Ogun water samples.

\begin{tabular}{|c|c|c|c|c|c|c|c|c|c|c|}
\hline & $\mathrm{Ag}$ & $\mathrm{Fe}$ & $\mathrm{Mn}$ & $\mathrm{Pb}$ & $\mathrm{Ni}$ & $\mathrm{Mg}$ & $\mathrm{Cd}$ & $\mathrm{Zn}$ & $\mathrm{Na}$ & $\mathrm{K}$ \\
\hline $\mathrm{Ag}$ & $\begin{array}{c}1.000 \\
(1.000)\end{array}$ & $\begin{array}{c}0.67 \\
(0.11)\end{array}$ & $\begin{array}{c}-0.55 \\
(-0.84)\end{array}$ & $\begin{array}{c}-0.39 \\
(-0.58)\end{array}$ & $\begin{array}{c}-0.36 \\
(-0.76)\end{array}$ & $\begin{array}{c}0.09 \\
(0.41)\end{array}$ & $\begin{array}{l}-0.12 \\
(0.02)\end{array}$ & $\begin{array}{c}-0.41 \\
(-0.30)\end{array}$ & $\begin{array}{l}-0.44 \\
(0.99)\end{array}$ & $\begin{array}{c}0.06 \\
(0.99)\end{array}$ \\
\hline $\mathrm{Fe}$ & & $\begin{array}{c}1.000 \\
(1.000)\end{array}$ & $\begin{array}{l}-0.17 \\
(0.42)\end{array}$ & $\begin{array}{c}0.07 \\
(-0.06)\end{array}$ & $\begin{array}{c}0.15 \\
(0.52)\end{array}$ & $\begin{array}{c}-0.05 \\
(-0.44)\end{array}$ & $\begin{array}{c}0.01 \\
(-0.55)\end{array}$ & $\begin{array}{c}-0.03 \\
(-0.68)\end{array}$ & $\begin{array}{c}-0.06 \\
(-0.56)\end{array}$ & $\begin{array}{c}-0.15 \\
(-0.50)\end{array}$ \\
\hline $\mathrm{Mn}$ & & & $\begin{array}{c}1.000 \\
(1.000)\end{array}$ & $\begin{array}{c}0.85 \\
(0.39\end{array}$ & $\begin{array}{c}0.82 \\
(0.95)\end{array}$ & $\begin{array}{c}-0.30 \\
(-0.50)\end{array}$ & $\begin{array}{c}-0.14 \\
(-0.41)\end{array}$ & $\begin{array}{c}0.80 \\
(-0.17)\end{array}$ & $\begin{array}{c}0.54 \\
(-0.93)\end{array}$ & $\begin{array}{c}-0.08 \\
(-0.97)\end{array}$ \\
\hline $\mathrm{Pb}$ & & & & $\begin{array}{c}1.000 \\
(1.000)\end{array}$ & $\begin{array}{c}0.99 \\
(0.57)\end{array}$ & $\begin{array}{c}-0.30 \\
(-0.41)\end{array}$ & $\begin{array}{l}-0.11 \\
(0.66)\end{array}$ & $\begin{array}{c}0.75 \\
(0.42)\end{array}$ & $\begin{array}{c}0.44 \\
(-0.44)\end{array}$ & $\begin{array}{c}-0.12 \\
(-0.48)\end{array}$ \\
\hline $\mathrm{Ni}$ & & & & & $\begin{array}{c}1.000 \\
(1.000)\end{array}$ & $\begin{array}{l}-0.27 \\
(0.60)\end{array}$ & $\begin{array}{c}-0.099 \\
(0.21)\end{array}$ & $\begin{array}{c}0.76 \\
(0.10)\end{array}$ & $\begin{array}{c}0.47 \\
(0.86)\end{array}$ & $\begin{array}{l}-0.13 \\
(0.84)\end{array}$ \\
\hline $\mathrm{Mg}$ & & & & & & $\begin{array}{c}1.000 \\
(1.000)\end{array}$ & $\begin{array}{c}-0.21 \\
(-2.08)\end{array}$ & $\begin{array}{l}-0.16 \\
(0.28)\end{array}$ & $\begin{array}{c}0.63 \\
(0.97)\end{array}$ & $\begin{array}{c}0.78 \\
(0.93)\end{array}$ \\
\hline $\mathrm{Cd}$ & & & & & & & $\begin{array}{c}1.000 \\
(1.000)\end{array}$ & $\begin{array}{l}-0.11 \\
(0.68)\end{array}$ & $\begin{array}{c}0.00 \\
(0.64\end{array}$ & $\begin{array}{c}-0.13 \\
(0.53\end{array}$ \\
\hline $\mathrm{Zn}$ & & & & & & & & $\begin{array}{c}1.000 \\
(1.000)\end{array}$ & $\begin{array}{c}0.53 \\
(0.64)\end{array}$ & $\begin{array}{l}-0.07 \\
(0.39)\end{array}$ \\
\hline $\mathrm{Na}$ & & & & & & & & & $\begin{array}{c}1.000 \\
(1.000)\end{array}$ & $\begin{array}{c}0.31 \\
(0.64)\end{array}$ \\
\hline $\mathrm{K}$ & & & & & & & & & & $\begin{array}{c}1.000 \\
(1.000)\end{array}$ \\
\hline
\end{tabular}

Note: Values in parentheses are for Ogun samples. 
than the values reported for this study [36] (Table 1). Whereas, chloride and sulphate have no stated WHO guideline values, while for nitrate it is $50 \mathrm{mg} / \mathrm{L}$ [37]. Kokoro Abu and Ogudu samples exceeded this guideline value, $50.83 \pm 1.46 \mathrm{mg} / \mathrm{L}$ (Kokoro Abu) and $73.18 \pm 24.31 \mathrm{mg} / \mathrm{L}$ (Ogudu) respectively. This is an indication that nitrate contamination is not widespread in the Lagos-Ogun axis groundwater. The primary health problem associated with high intake of $\mathrm{NO}_{3}^{-}$in drinking water is methemoglobinemia (blue baby syndrome) (Gupta et al. 2000). However, the adult individuals can tolerate high levels of $\mathrm{NO}_{3}^{-}$with little or no documented adverse health effects as posited by Bruning-Fann and Kaneene, 1993 [11]. The levels of $\mathrm{Na}$ and $\mathrm{K}$ in the water samples (Table 2) are lower than the reported global background values $(\mathrm{mg} / \mathrm{L})$ of $5.70-599.00(\mathrm{Na})$ and $1.60-15.00$ (K) respectively. $\mathrm{Na}$ and $\mathrm{K}$ are essential elements for human nutrition; they maintain the normal osmotic pressure in cells. USEPA limit for Na in drinking water is $20 \mathrm{mg} / \mathrm{L}$. There are scientific evidences linking excessive sodium intake to age-related increases in blood pressure leading to hypertension [38].

The concentrations of $\mathrm{Mn}, \mathrm{Pb}, \mathrm{Ni}$ and $\mathrm{Cd}$ were compared with WHO guidelines [37] for drinking water (Table 2). All water samples are within the acceptable guideline values of $0.4 \mathrm{mg} / \mathrm{L}(\mathrm{Mn}), 0.003 \mathrm{mg} / \mathrm{L}(\mathrm{Cd})$ and $0.07 \mathrm{mg} / \mathrm{L}(\mathrm{Ni})$ as recommended by WHO. However, nickel value at Akesan 1 and Lusada $2(0.09 \mathrm{mg} / \mathrm{L})$ were than the WHO value. The concentration of $\mathrm{Pb}$ in Erunwen 1, Akesan 1 and Akesan 2 exceed the permissible limit of $0.01 \mathrm{mg} / \mathrm{L}$ by WHO.

The other metals have no WHO guideline values but compares favourably with other reported global background values (mg/L): 0.00094 - $0.067(\mathrm{~Pb}), 0.00065$ - $0.0156(\mathrm{Ni}), 0.00017$ - $0.00023(\mathrm{Cd}), 0.0069$ - $0.302(\mathrm{Zn})$ for Ubon Ratchathani province of Thailand; 0.12 - 0.72 (Fe), 0.028 - $0.20(\mathrm{Mn}), 0.053$ - $0.70(\mathrm{~Pb}), 0.032$ - 0.24 (Ni), ND - 0.017 (Cd), 0.078 - 2.499 (Zn) for Tigray region, Northern Ethiopia; 0.0016 - 0.347 (Fe), ND - 0.113 (Mn), ND - $0.0088(\mathrm{~Pb}), \mathrm{ND}-0.013(\mathrm{Ni}), \mathrm{ND}-0.0015$ (Cd), 0.0003 - 0.32 (Zn) for Kermanshah, Iran; 0.002 0.57 (Fe), 0.005 - 0.02 (Mn), 0.00 - 0.04 (Pd), 0.00 - 0.002 (Cd), 0.00 - 0.047 (Zn) for Ejisu-Juaben municipality, Ghana; 2.42 - 2.675 (Fe), 0.51 - $0.67(\mathrm{Mn}), 0.11$ - 0.29 (Pb), 0.025 - $0.065(\mathrm{Ni}), 0.015$ - 0.03 (Cd), 0.35 - 0.59 (Zn) for Eket and Esit Eket, Nigeria; 0.11 - 24.2 (Fe), 0.06 - 16.00 (Mn), 2.00 - 70.00 (Mg), ND - 0.2 (Cd), 5.70 - $599.00(\mathrm{Na}), 1.60$ - $15.00(\mathrm{~K})$ for Bangladesh; 0.0007 - $0.01(\mathrm{~Pb}), 0.00013$ - $0.00050(\mathrm{Cd})$ for Shizuishan city, Northern China. The $\mathrm{Pb}$ values (ND - $0.35 \mathrm{mg} / \mathrm{L}$ ) obtained in this study were higher than most recorded global background values with the exception of the values obtained in Ethiopia, Lao PDR and Swat, Northern Pakistan $(0.015-0.075 \mathrm{mg} / \mathrm{L})$. The zinc and iron concentrations obtained in this study $(0.001-0.082 \mathrm{mg} / \mathrm{L})$ and $(0.01-$ $0.012 \mathrm{mg} / \mathrm{L}$ ) respectively were below the values reported for Ethiopia, Thailand, Iran, Ghana, India and Bangladesh respectively [9] [17] [21]-[25].

Kempster and co-workers [13] are of the opinion that no undesirable health or aesthetic effects have been shown to be associated with magnesium concentrations in drinking water of $<70 \mathrm{mg} / \mathrm{L}$. However, Grimason et al., 2013 [36] opined that levels $>30 \mathrm{mg} / \mathrm{L}$ may impair the lathering of soap, while concentrations ranging from $70-100 \mathrm{mg} / \mathrm{L}$ may impart a bitter taste to the water and cause diarrhoea in sensitive users including infants [39]. At levels above $100 \mathrm{mg} / \mathrm{L}$ the bitter taste and diarrhoea may be exacerbated. Studies have equally suggested that dissolved metals in drinking water such as iron, magnesium and calcium may contribute significantly to the dietary intakes of these metals thus reducing the risk of anaemia, cardiovascular and osteoporosis respectively [40].

The MPI shown in Table 2 reveal values ranging from 0.01 to $0.11 \mathrm{mg} / \mathrm{L}$. These values are less than the baseline value of $1 \mathrm{mg} / \mathrm{L}$ reported earlier [41].

The t-test statistical analysis for the physico-chemical parameters recorded between the Lagos and Ogun States samples indicate non-significant differences ranging from 0.49 to $1.70\left(\mathrm{t}_{\mathrm{cal}}\right)$ except for dissolved oxygen which recorded a significant difference of $2.34\left(\mathrm{t}_{\mathrm{cal}}\right)$ against a $\mathrm{t}_{\mathrm{tab}}$ value of 2.10. These values indicate that contamination by metals and the levels of other physico-chemical parameters are not State dependent. Strong positive correlation existed between the metals in most of the sample sites, thus suggesting that the metals are likely to have originated from similar sources (Table 5 and Table 6).

The non-cancer health quotient for the study area for both adult and children is shown in Table 3. Whereas, the carcinogenic risk assessment values for Erunwen 3 sampling site are $2.51 \mathrm{E}-02$ and $5.75 \mathrm{E}-02$ for adult and children respectively, the other values are shown in Figure 2.

The non-cancer HQs of all eight metals in Lagos, Ogun for both adult and children were below the recommended HQ threshold of 1, indicating no immediate significant adverse health effects from the groundwater consumption in most of the sampling areas. However, long-term daily consumption of the groundwater in Erunwen 3 could cause chronic negative health effects since the maximum HI values for ingestion were 17.9 and 8.11 for children and adult respectively which are far greater than 1 . 
Only Cd, among the metals analyzed in this study area is classified as a known human carcinogen by the International Agency for Research on Cancer and the Integrated Risk Information System of United States Environmental Protection Agency [42].

The quanta of potential exposure to carcinogenic risk in the study area are (\%): Negligible ( $<1 \mathrm{E}-06), 50$ and 55 respectively for adults and children; Acceptable (1E-06 to 1E-04), 45 and 40, adults and children respectively; Excessive (>1E-04), 5 for both adult and children.

Following the scheme proposed by Kempster et al. [13] on the classification of drinking water quality (Table 4), Eyita 3 is in Class 0 (ideal and suitable for lifetime use). However, water sourced from Erunwen 1 and 2, Agbele, Eyita 1 and 2, Akesan 2, Ilado, Ogijo and Magboro fall into Class I, i.e. water safe for lifetime use but falls short of the ideal water quality in that there may be possible exposure to health hazards. Slightly acidic $\mathrm{pH}$ values and relatively high nitrate, $\mathrm{Fe}, \mathrm{Mn}$ and $\mathrm{Cd}$ values are responsible for these water samples falling short of the ideal range of values [36]. Similarly, borehole water samples collected from Oshodi, Akesan 1, Ilupeju-Ag- bara, Lusada 1 and 2 are in Class II, which is water that may result in adverse health effects with prolonged use over many years or with lifetime use. Nonetheless, water samples from five locations i.e. Erunwen 3, Kokoro Abu, Ikosi-Ketu, Ogudu and Ibafo are in Class III , water not suitable for use as drinking water without adequate treatment to shift it to lower and safer Class. It should be noted that Ibafo fell into class III because of its relatively high nitrate level of $24.43 \pm 7.74 \mathrm{mg} / \mathrm{L}$, whose source appears to be diffuse [43] [44].

\section{Conclusion}

It is concluded among others, that improper disposal of solid waste and sewage, may have contaminated the ground water in these communities with nitrate and metals such as $\mathrm{Ag}, \mathrm{Mn}, \mathrm{Pb}$, Ni and Cd. Inter-metal correlation showed a strong correlation between metal pairs. The computation of non-cancer HQs of selected metals in Lagos and Ogun sampling locations for both adult and children were below the recommended HQ threshold of 1 . This is an indication that there are no immediate significant adverse health effects arising from the consumption of groundwater in most of the sampling locations. However, water sourced Erunwen 3, Kokoro Abu, Ikosi-Ketu, Ogudu and Ibafo are of Class III, i.e. water not suitable for drinking without adequate treatment. The water classification system adopted here is incisive making it imperative for proper water quality monitoring and management in these areas. Emphasis should be on municipal and community based water supply. It is also recommended that mitigation measures be conducted in the areas at risk, with the particular aim of lowering the concentrations of $\mathrm{Ag}, \mathrm{Mn}, \mathrm{Pb}, \mathrm{Ni}, \mathrm{Mg}$ and $\mathrm{Cd}$ to meet WHO drinking water standards. Public health awareness programs should be conducted by relevant government and non-governmental agencies to raise consciousness about the adverse health consequences of consuming metal contaminated water above recommended limits.

\section{Acknowledgements}

The authors are grateful to Lagos State Environmental Protection Agency (LASEPA) and Dr. Wale Raimi, University of Dundee, Scotland, for valuable assistance. We are equally grateful to the families whose households were used for this study.

\section{Ethical Statement}

The work has not been published before and it's not under consideration anywhere else. Authors approve of the submission to Journal Water Resource and Protection. We affirm that it is an original work and there is no conflict of interest.

\section{References}

[1] Chowdary, V.M., Rao, N. and Sarma, P.B.S. (2005) Decision Support Framework for Assessment of Non-Point-Source Pollution of Groundwater in Large Irrigation Projects. Agricultural Water Management, 75, 194-225. http://dx.doi.org/10.1016/j.agwat.2004.12.013

[2] Chanpiwat, P., Lee, B., Kim, K. and Sthiannopkao, S. (2014) Human Health Risk Assessment for Ingestion Exposure to Groundwater Contaminated by Naturally Occurring Mixtures of Toxic Heavy Metals in the Lao PDR. Environmental Monitoring and Assessment, 186, 4905-4923. http://dx.doi.org/10.1007/s10661-014-3747-0

[3] Rivett, U., Champanis, M. and Wilson-Jones, T. (2013) Monitoring Drinking Water Quality in South Africa: Designing Information Systems for Local Needs. Water SA, 39, 409-414. http://dx.doi.org/10.4314/wsa.v39i3.10 
[4] Khan, S., Shahnaz, M., Jehan, N., Rehman, S., Shah, M.T. and Din, I. (2013a) Drinking Water Quality and Human Health Risk in Charsadda District, Pakistan. Journal of Cleaner Production, 60, 93-101. http://dx.doi.org/10.1016/j.jclepro.2012.02.016

[5] Adeniyi, A., Osifeko, O., Owoade, O., Omotayo, Y., Ajede, E., Ibrahim, A. and Balogun, R. (2014) Metal Burden as Template for Assessing the Quality of Raw Water Sourced from Two Rivers by Lagos State Water Corporation, Nigeria. In: Bhowon, M.G., Jhaumeer-Laullo, S., Wah, H.L. and Ramasami, P., Eds., Chemistry: The Key to our Sustainable Future, Springer, Dordrecht, 163-172. http://dx.doi.org/10.1007/978-94-007-7389-9_12

[6] Soyingbe, A.A., Salako, S.G. and Hammed, T.B. (2014) Sanitary Condition and Characteristics of Borehole Water Samples in Ogun State College of Health Technology, Ilese-Ijebu, Nigeria. African Journal of Medical and Health Sciences, 1, 57-63.

[7] Knuppe, K. (2011) The Challenges Facing Sustainable and Adaptive Groundwater Management in South Africa. Water SA, 37, 67-79. http://dx.doi.org/10.4314/wsa.v37i1.64110

[8] Emmanuel, E., Pierre, M.G. and Perrodin, Y. (2009) Groundwater Contamination by Microbiological and Chemical Substances Released from Hospital Wastewater and Health Risk Assessment for Drinking Water Consumers. Environment International, 35, 718-726. http://dx.doi.org/10.1016/j.envint.2009.01.011

[9] Muhammad, S., Shah, M.T. and Khan, S. (2011) Health Risk Assessment in Drinking Water and Source Appointment Using Multivariate Statistical Techniques in Kohistan Region, Northern Pakistan. Food and Chemical Toxicology, 48, 2855-2864. http://dx.doi.org/10.1016/j.fct.2010.07.018

[10] Grimason, A.M., Morse, T.D., Beattie, T.K., Masangwi, S.J., Jabu, G.C., Taulo, S.C. and Lungu, K.K. (2013) Classification and Quality of Groundwater Supplies in the Lower Shire Valley, Malawi-Part 1: Physico-Chemical Quality of Borehole Water Supplies in Chikhwawa, Malawi. Water SA, 39, 563-572. http://dx.doi.org/10.4314/wsa.v39i4.16

[11] Bruning-Fann, C.S. and Kaneene, J.B. (1993) The Effects of Nitrate, Nitrite, and N-Nitroso Compounds on Human Health: A Review. Veterinary and Human Toxicology, 35, 521-538.

[12] WHO (1996) Guidelines for Drinking-Water Quality, Vol. 2: Health Criteria and Other Supporting Information. 2nd Edition, World Health Organization, Geneva.

[13] Kempster, P.L., Van Vliet, H.R. and Kuhn, A. (1997) The Need for Guidelines to Bridge the Gap between Ideal Drinking-Water Quality and That Quality Which Is Practically Available and Acceptable. Water SA, 23, 163-167.

[14] Gupta, S.K., Gupta, R.C., Seth, A.K., Gupta, A.B., Bassin, J.K. and Gupta, A. (2000) Recurrent Acute Respiratory Tract Infections in Areas with High Nitrate Concentrations in Drinking Water. Environmental Health Perspectives, 108, 363-366. http://dx.doi.org/10.1289/ehp.00108363

[15] Khan, K., Lu, Y., Khan, H., Zakir, S., Ihsanullah, Khan, S., Khan, A.A., Wei, L. and Wang, T. (2013) Health Risks Associated with Heavy Metals in the Drinking Water of Swat, Northern Pakistan. Journal of Environmental Sciences, 25, 2003-2013. http://dx.doi.org/10.1016/S1001-0742(12)60275-7

[16] Ho, K.C., Chow, Y.L. and Yau, J.T.S. (2003) Chemical and Microbiological Qualities of the East River (Dongjiang) Water, with Particular Reference to Drinking Water Supply in Hong Kong. Chemosphere, 52, 1441-1450. http://dx.doi.org/10.1016/S0045-6535(03)00481-8

[17] Rajmohan, N. and Elango, L. (2005) Distribution of Iron, Manganese, Zinc and Atrazine in Groundwater in Parts of Pala and Cheyyar River Basins, South India. Environmental Monitoring and Assessment, 107, 115-131. http://dx.doi.org/10.1007/s10661-005-5307-0

[18] Leung, C.M. and Jiao, J.J. (2006) Heavy Metal and Trace Element Distributions in Groundwater in Natural Slopes and Highly Urbanized Spaces in Mid-Levels Area, Hong Kong. Water Research, 40, 753-767. http://dx.doi.org/10.1016/j.watres.2005.12.016

[19] Mebrahtu, G. and Zerabruk, S. (2011) Concentration of Heavy Metals in Drinking Water from Urban Areas of the Tigray Region, Northern Ethiopia. Momona Ethiopian Journal of Science, 3, 105-121. http://dx.doi.org/10.4314/mejs.v3i1.63689

[20] Li, P.Y. and Qian, H. (2011) Human Health Risk Assessment for Chemical Pollutants in Drinking Water Source in Shizuishan City, Northwest China. Iranian Journal of Environmental Health Science and Engineering, 8, 41-48.

[21] Kolo, B.G. and Waziri, M. (2012) Determination of Some Heavy Metals in Borehole Water Samples of Selected Motor Parks in Maiduguri, Nigeria. International Journal of Basic and Applied Chemical Sciences, 2, 18-20.

[22] Pirsaheb, M., Khosravi, T., Sharafi, K., Babajani, L. and Rezaei, M. (2013) Measurement of Heavy Metals Concentration in Drinking Water from Source to Consumption Site in Kermanshah-Iran. World Applied Sciences Journal, 2, 416-423.

[23] Wongsasuluk, P., Chotpantarat, S., Siriwong, W. and Robson, M. (2014) Heavy Metal Contamination and Human Health Risk Assessment in Drinking Water from Shallow Groundwater Wells in an Agricultural Area in Ubon Ratchathani Province, Thailand. Environmental Geochemistry and Health, 36, 169-182. 
[24] Towfiqul-Islam, A.R.M., Rakib, M.A., Saidul-Islam, M., Jahan, K. and Patwary, M.A. (2015) Assessment of Health Hazard of Metal Concentration in Groundwater of Bangladesh. American Chemical Science Journal, 5, 41-49. http://dx.doi.org/10.9734/ACSJ/2015/13175

[25] Boateng, T.K, Opoku, F., Acquaah, S.O. and Akoto, O. (2015) Pollution Evaluation, Sources and Risk Assessment of Heavy Metals in Hand-Dug Wells from Ejisu-Juaben Municipality, Ghana. Environmental Systems Research, 4, 18-30. http://dx.doi.org/10.1186/s40068-015-0045-y

[26] Udousoro, I.I. and Udoh, V.M. (2015) Source Identification and Health Risks Assessment of Toxic Metals in Rainwater and Groundwater in Eket and Esit Eket, Nigeria. American Scientific Research Journal for Engineering, Technology, and Sciences, 13, 227-245.

[27] American Public Health Association (APHA) (1998) Standard Methods for the Examination of Water and Wastewater. APHA AWWA WPCE, 20th Edition, Byrd Progress, Springfield.

[28] Usero, J., González-Regalado, E. and Graccia, I. (1997) Trace Metals in the Bivalve Molluscs Ruditapes decussatus and Ruditapes philippinarum from the Atlantic Coast of Southern Spain. Environment International, 23, 291-298. http://dx.doi.org/10.1016/S0160-4120(97)00030-5

[29] Adeniyi, A.A., Yusuf, K.A. and Okedeyi, O.O. (2008) Assessment of the Exposure of Two Fish Species to Metals Pollution in the Ogun River Catchments, Ketu, Lagos, Nigeria. Journal of Environmental Monitoring and Assessment, 137, 451-458. http://dx.doi.org/10.1007/s10661-007-9780-5

[30] Shah, M.T., Begum, S. and Khan, S. (2010) Pedo and Biogeochemical Studies of Mafic and Ultramafic Rocks in the Mingora and Kabal Areas, Swat, Pakistan. Environmental Earth Sciences, 60, 1091-1102. http://dx.doi.org/10.1007/s12665-009-0253-8

[31] Chen, J. (2002) Analysis of Water Environment in the Xinjiang Arid Region. Arid Environmental Monitoring, 16, 223-227.

[32] WHO (2008) Guidelines for Drinking-Water Quality. 3rd Edition, World Health Organization, Geneva.

[33] United States Environmental Protection Agency (USEPA) (1989) Risk Assessment Guidance for Superfund, Vol. 1: Human Health Evaluation Manual (Part A). EPA/540/1-89/002, Interim Final, Office of Emergency and Remedial Response, Washington DC.

[34] United States Environmental Protection Agency (USEPA) (2000) Supplementary Guidance for Conducting Health Risk Assessment of Chemical Mixtures. EPA/630/R-00/002, Risk Assessment Forum, Washington DC.

[35] United States Environmental Protection Agency (USEPA) (2011) Exposure Factors Handbook 2011 Edition (Final). United States Environmental Protection Agency, Washington DC.

[36] Grimason, A.M., Beattie, T.K., Morse, T.D., Masangwi, S.J., Jabu, G.C., Taulo, S.C. and Lungu, K.K. (2013) Classification and Quality of Groundwater Supplies in the Lower Shire Valley, Malawi-Part 2: Classification of Borehole Water Supplies in Chikhwawa, Malawi. Water SA, 39, 573-581. http://dx.doi.org/10.4314/wsa.v39i4.17

[37] WHO (2011) Guidelines for Drinking-Water Quality. 4th Edition, World Health Organization, Geneva.

[38] United States Environmental Protection Agency (USEPA) (2003) Drinking Water Advisory: Consumer Acceptability Advice and Health Effects Analysis of Sulfate. United States Environmental Protection Agency, Washington DC.

[39] WHO (2009) The Mineral Composition of Water and Its Contribution to Calcium and Magnesium Intake. World Health Organization, Geneva.

[40] WHO (2003) Total Dissolved Solids in Drinking Water: Background Document for Development of WHO Guidelines for Drinking Water Quality. WHO/SDE/WSH/03.04/16, World Health Organization, Geneva.

[41] Mmolawa, K.B., Likuku, A.S. and Gaboutloeloe, G.K. (2011) Assessment of Heavy Metal Pollution in Soils along Major Roads in Botswana. African Journal of Environmental Science and Technology, 5, 186-196.

[42] International Agency for Research on Cancer (IARC) (2011) IARC Monographs on the Evaluation of Carcinogenic Risks to Humans, Supplement 7: Overall Evaluations of Carcinogenicity: An Update of IARC Monographs Volumes $1-42$.

[43] Liu, A.G., Ming, J.H. and Ankumah, R.O. (2005) Nitrate Contamination in Private Wells in Rural Alabama, United States. Science of the Total Environment, 346, 112-120. http://dx.doi.org/10.1016/j.scitotenv.2004.11.019

[44] Novotony, V. and Olem, H. (1994) Water Quality: Prevention, Identification and Management of Diffuse Pollution. Van Nostrand Reinhold, New York, 18-25. 